\title{
Organizational Development within the Government Sector: An Innovative Strategic Framework
}

\author{
Ali M. Al-Khouri ${ }^{1, *}$ \\ ${ }^{1}$ British Institute of Technology and E-commerce, London, UK \\ *Correspondence: British Institute of Technology and E-commerce, London, UK. E-mail: alkhouriali@gmail.com
}

Received: November 14, 2014

Accepted: April 20, $2015 \quad$ Online Published: July 7, 2015

doi:10.5430/mos.v2n3p40

URL: http://dx.doi.org/10.5430/mos.v2n3p40

\begin{abstract}
Today's business world is more complex and fragmented with new underlying terms and concepts. As such, organizations are forced to understand the dynamics shaping today's fuzzy world. Government organizations amid all this have been facing turbulent times to fulfill their objectives. Existing research that provides qualitative cases from governments' fields of practice is limited and narrowed in scope that is either of academic or commercial orientation. This article explores and presents a case study of an innovative strategic organizational development framework implemented at one of the most successful organizations in the Middle East, which is also internationally renowned for best practices in management and technology implementations. The framework demonstrates the overall organizational factors that have been considered as key drivers for organizational performance and excellence. Its primary contribution is to highlight the benefits of having the organization's strategy as the major driver for the selection and implementation of different management tools and processes to improve organizational performance and excellence. The framework represents a structured approach for the development of key management systems to accelerate the execution of a long-term strategic plan and the attainment of its expected outcomes, highlighting essential focus areas required to maximize organizational effectiveness and maturity. It also serves as a guide for organizations in developing and/or enhancing existing organizational management practices.
\end{abstract}

Keywords: Organizational development, Strategic planning, Strategic framework, Organizational excellence, Public sector strategies

\section{Introduction}

Organizations today face an ever-changing landscape of macroeconomic realities (Accenture, 2013). Industry trends and internal challenges are forcing organizations to radically rethink their business and operating models to be better aligned with the changing world and capitalize on value-generation opportunities (ibid). Among the many approaches to address such challenges is the application of strategic planning and development. From a macro perspective, the formal appearance of strategies in businesses was in the early ' $60 \mathrm{~s}$. The strategies focused on needs related to survival in the face of tough competition and were driven by profit needs amid rising operational costs.

Strategy in governments is a recent phenomenon though and has become an important fundament to address the changing dynamics of governance in the government business. However, research studies of the past 30 years revealed that more than two-thirds of strategies fail (Branston et al., 2009; Chapman, 2002; Devarajan and Kanbur, 2012; Heeks, 2003; Winston, 2006). In fact, others who have investigated strategy implementations found this to be more alarming, with an 80-90\% failure rate. Another study found that from 2000 to 2010, less than half of U.S. start-ups lasted more than 3 years (Cespedes et al., 2013). Fewer than $6 \%$ of surveyed organizations reached more than $\$ 10$ million in revenue by 2010 (ibid.).

Overall, the literature showed that corporate history is fraught with stories of failure. While reasons abound, failures are a fact, faced both in the corporate and the government sectors. There are many reasons behind such failure stories. Figure 1 depicts survey results of a study carried out in Europe that is very relevant to both the public- and private-sector organizations even today. Decades of research reports that most organizations fail at strategy implementation (Mass, 2014). 


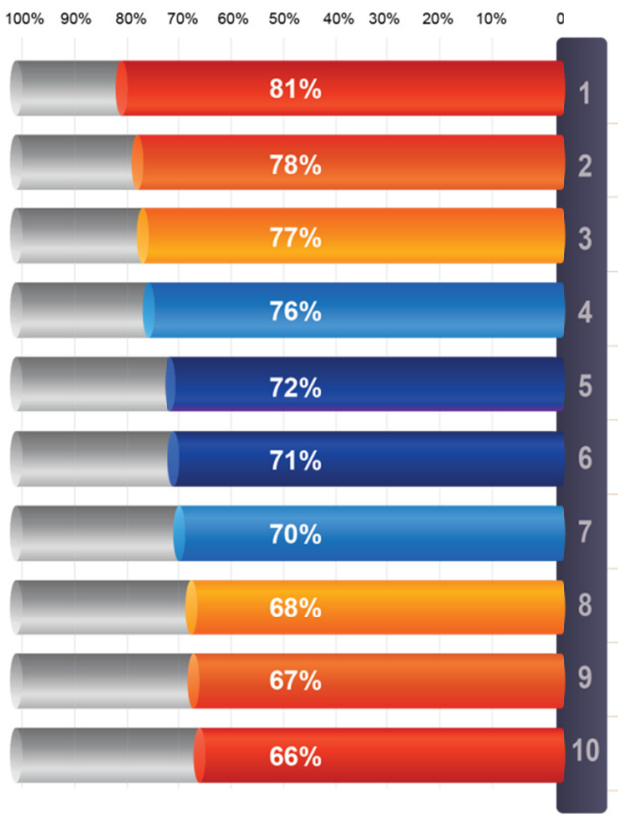

\section{Execution (Poor alignment - synchronization)}

Resist the change

Not communicated to all the people involved

The plan is vague (poor communication)

The goal isn't compelling

Lack of team Involvement

Too many goals

The goal isn't clear

The cultural implications are overlooked

The correct metrics aren't chosen

Figure 1. Top 10 Reasons Why Strategies Fail

Source: (HRN-Europe, 2009)

We tend to argue that failure is more predominant in the public sector when compared to the private sector. Of course, government organizations do not go bankrupt, but the consequences of organizational failure have far-reaching impact. Private sector by nature is dynamic and more adaptive to change. Public sector, on the contrary, is more bureaucratic and hence slower to change. According to researchers, there are many factors that contribute to organizational strategy failure in governments. These are related to:

- Lack of acceptance to change;

- Inability to see trends;

- Lack of understanding of citizen needs;

- Lack of skilled resources;

- Lack of inter-agency coordination;

- Lack of effective communication; and

- Political instability.

Thus, many researchers have argued that organizations need to have a more holistic developmental approach to deliver sustainable performance (Beckhard, 1969; Child, 2005; Cummings and Worley, 2001; French and Bell, 1973; Margulies, 1972; Rothwell et al., 2010; Western, 2010). This is referred to in the literature as organization development. Organization development is concerned with a deliberately planned, organization-wide change, uses behavioral science knowledge, targets human and social process of organizations, and intends to build the capacity to adapt and renew organizations (Cummings and Worley, 2001).

Organization design theorists and practitioners believe that every part of an organization is integral to a system that relies on and impacts other elements of the internal and external environment in which the organization operates. Thus, they see organizational development to deal with a wide range of organizational systems such as: organization diagnostic, evaluation, strategic thinking, culture change, change management, coaching, mentoring, leadership development, team building, organizational design, performance management, talent management, human resources processes, learning and development, effectiveness, etc. This is overwhelming indeed!

There are hundreds of written books and thousands of articles that have examined organizational development in different contexts and provided various management techniques and approaches to select and apply. However, a key observation is that current literature is either written from purely academic perspectives or is commercially driven. Besides, the existing body of knowledge has very few examples of case studies from public-sector organizations. 
This article attempts to add to the limited knowledge repository in this critical field of practice by exploring and presenting a case study of an innovative strategic organizational framework implemented at one of the most successful organizations in the Middle East, Emirates Identity Authority (Emirates ID), which is also considered as a renowned international benchmark for best practices both in management and technology implementations. The framework demonstrates the overall organizational factors that have been considered as key drivers for organizational performance and excellence at Emirates ID.

The primary contribution of the framework is argued to highlight the benefits of having the organization's strategy as the major driver for the selection and implementation of different management tools and processes to improve organizational performance and excellence. The primary framework represents a structured approach for the development of key management systems to accelerate the execution of a long-term strategic plan and the attainment of its expected outcomes, highlighting essential focus areas required to maximize organizational effectiveness and maturity. It also serves as a guide for organizations in developing and/or enhancing existing organizational management practices.

This article is structured as follows. In section 2, the methodology followed in this research is described. In section 3, background information about Emirates ID is provided. Section 4 represents the main body of this article where the organizational strategic framework is presented and discussed. Section 5 depicts the strategic results achieved by Emirates ID after the implementation of the framework. The article is then concluded in section 6.

\section{Research Methodology}

Organizations today are driven by strategy. In this context, the purpose of this study was to (1) explore the literature to understand the availability and role of strategy management in organizational development and (2) describe how a strong strategy management framework is implemented in government organization development.

Action Research methodology is primarily adopted in this study, leveraging the author's position as the head of the organization used for the case study and the author's experience of guiding the organization through three successful strategy cycles. This is complemented with the case study as an example to demonstrate the findings. It is a characteristic of action research that the participants or 'practitioners' as well as the researchers participate in the analysis, design, and implementation processes and usually add as much as the researchers to any decision-making (Harrison and Callan, 2013). As a close participant in the strategy formulation and the monitoring of the strategy implementation, the author has had the opportunity to continually test the framework - change, update and fine tune it — so that the test and the research questions are developed.

This research is intended to provide new data and information for the practitioners from first-hand experience, where the researcher studies the environment not as an independent outside observer, but as an insider and part of the action. This is then presented as a case study as an observer, providing the best of two methodologies for objective presentation. The contextual nature of the case study is illustrated in Yin's definition as an empirical inquiry that "investigates a contemporary phenomenon within its real-life context and addresses a situation in which the boundaries between phenomenon and context are not clearly evident" (Yin, 1993).

As stated, the purpose of this article is to create a better understanding of how strategy management is being pursued in government organizations for their development. This entailed conducting a detailed study of the context and the processes of implementation, as well as the change resulting from the implementation process. Such a focus led to the adoption of an interpretive stance, which seeks to uncover truth by understanding the phenomena in their real-life context (Walsham, 1995). The selected organization was one of the most successful government organizations in the United Arab Emirates (Emirates Identity Authority), especially considering the accessibility and proximity of the researcher within the organization.

Single case studies have been under criticism based on the fact there is little basis for scientific generalization. However, there are several rationales for a single case research. The first rationale for the single case is that it represents the critical case in testing a well-formulated theory and the second may be that a single case may represent an extreme or unique case that is worth documenting and analyzing, and the third rationale is the revelatory case: The revelatory case exists when a phenomenon not previously accessible to scientific investigation is revealed. This paper alludes to the third rationale of revelation.

The selection of the organization was based on two issues - one was accessibility and second was that it is a renowned organization of international repute with several awards in organizational excellence. The research design for this study is a descriptive and interpretive case study that is analyzed through qualitative methods. Data collection 
was done through both secondary and primary sources. Primary data sources included participation observation, group discussion, which provided face-to-face contact with the actors in order to explore and probe responses. Secondary data sources mainly covered publications and technical documentation analysis. Literature review provided an essential content preparation of this research article, which helped to provide an overview of the research field and practices and enabled cross-checks between the case study and literature findings.

\section{Emirates ID and Strategy: A Background Note}

Emirates Identity Authority (Emirates ID) is a federal government agency established in 2004 in the United Arab Emirates (UAE). The main objective of the organization was to develop a national identity management infrastructure that provides advanced mechanisms to identify and authenticate and thereby assert personal identity. This in turn is envisaged to contribute toward UAE government 2021 goals of enhancing national and individual security and digital economy development (e-government transformation and e-commerce).

Although the organization was constituted with a clear mandate and charter, it did not have an official operating strategy in the first two and a half years of operation, and the 2007-2010 strategy represented the first strategy cycle since its establishment. The strategy planning team in general assumed a smooth journey, but the execution was found to be bumpy. It was not until 2009 that the organization realized the strategy was not on track: it found that it had achieved less than $20 \%$ of its set objectives. After a change in higher management teams in mid-2009, the organization developed a new strategy with more ambitious goals and objectives.

The new management team derived a carefully developed strategic plan that incorporated different smart measures to ensure a smooth journey this time. However, the journey once again had many ups and downs and faced even more complex challenges. Despite all this, Emirates ID was able to fulfill its strategy and positioned itself as one of the most successful organizations in the country, with renowned international reputation in strategic planning and corporate excellence. Arguably, Emirates ID must have been following a prescription that was maintained and sustained throughout the design and execution of its organizational development strategy. We shall attempt to explore the framework adopted and discuss its components in the following section.

\section{The Strategic Framework at Emirates ID}

Let us examine this prescription that has won the organization such accolades. Figure 2 depicts an overview of the strategic framework developed and implemented at Emirates ID. In principle, the framework consists of three circles, each of which represents a set of functions. The inner circle (Tier 1) represents the primary performance dimensions, directly derived from the long-term strategic planning guidelines and balanced across the different (yet complementary) organizational perspectives. The middle circle (Tier 2) deals with key foundational capabilities that represent the backbone of the management approach, complemented by differentiation capabilities that have supported the organization to develop and sustain superior performance. The third circle (Tier 3) deals with dimensions related to communication and leadership, key success factors to the proper execution of the strategy.

As depicted in the framework, it has two guide handles: results and innovation. These two handles were seen as key enablers and the necessary rudders to keep the organization focused. The results handle represents the organizational culture at Emirates ID - an orientation that focused largely on outcomes with outputs tracked as milestones. "What do we want to accomplish by doing this? How can we make it happen? What do we have to do now to get it done?" These were simple questions that higher management tended to regularly ask. With such orientation, higher management teams tended to see the end result and always looked for the fastest way to get there. Not surprisingly, this represented the strongest handle that supported the organization to 'fly' toward the desired strategic results.

The other handle represents 'innovation' that basically fuelled the implementation journey to go the extra mile and accelerated the progress. Investment in innovation contributed to the realization of drastic improvement in efficiency, effectiveness, productivity, and overall differentiation and competitiveness. Innovation enabled the organization to see the implementation through a different lens. Emirates ID has put innovation at the center of its culture and as a means to constantly discover better ways of doing things; more productive, more responsive, more inclusive, and even more fun. (Note 1) (See also section 4.2.5). 


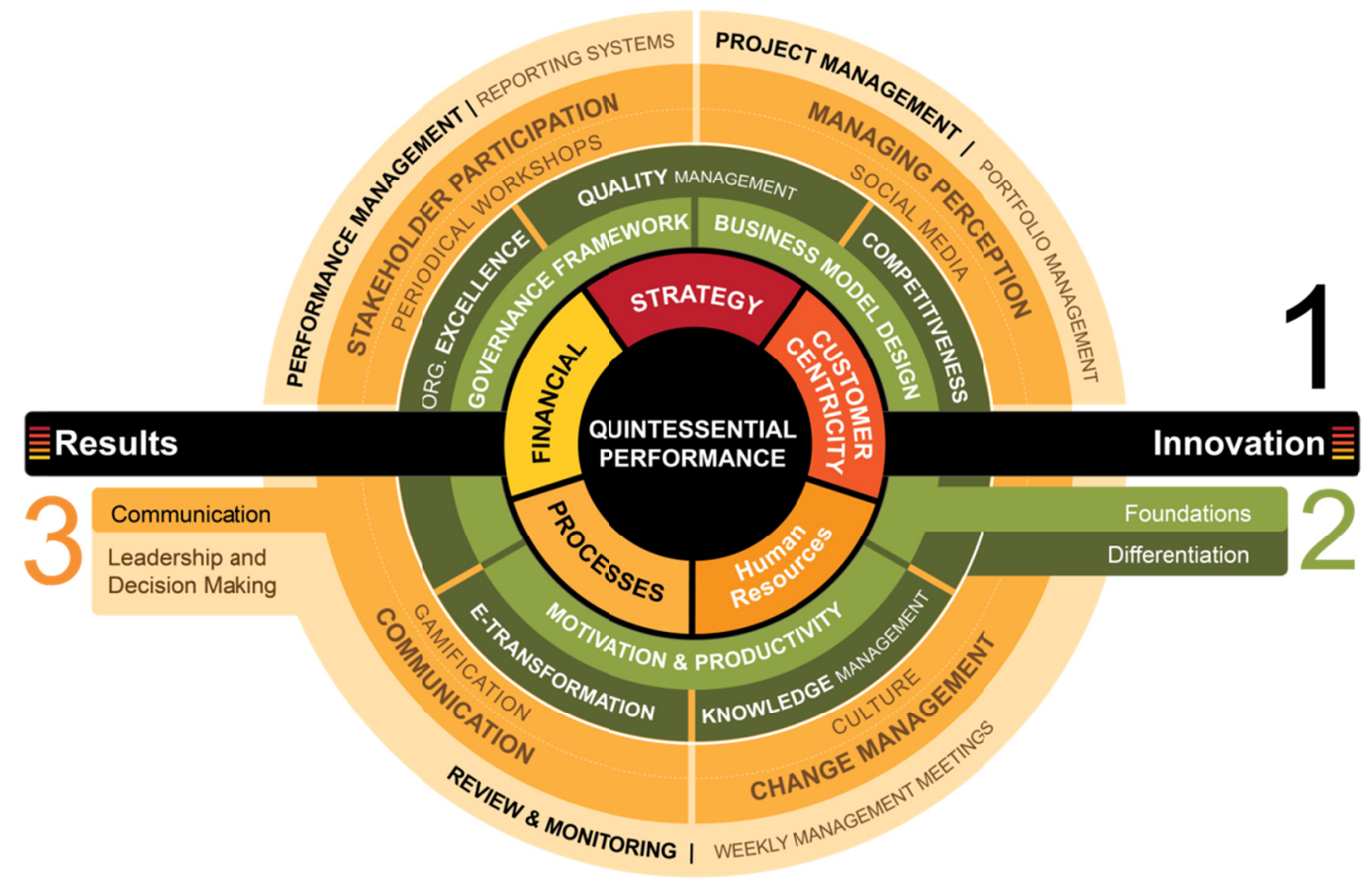

Figure 2. Strategic Framework - Architecture and Components

\subsection{Tier 1: Quintessential Performance Dimensions}

The inner circle ring of the framework represents key performance dimensions that Emirates ID used to continuously keep an eye on. See also Figure 3. They derived expected targets and guided the management in the overall strategy implementation. The use of a balanced scorecard facilitated the creation of the right parameters for such controls in the criteria selection, interpretation, and reporting of performance. Each of these dimensions will be discussed in the next sub-sections.

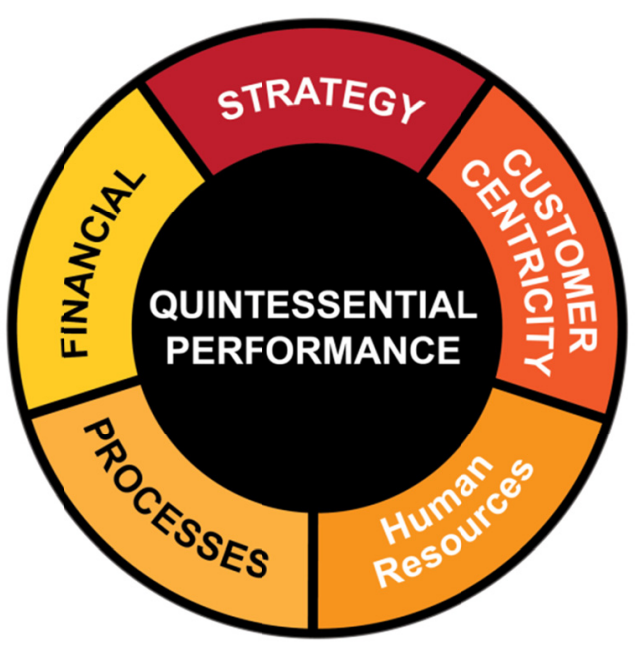

Figure 3. Key Performance Dimensions

\subsubsection{Core Strategy Development: The Starting Point}

The articulation of Emirates ID's 2010-2013 strategy followed a systematic and formal development process, 
including the preparation of many analytical and review studies to provide in-depth understanding of the Emirates ID's potential for further development. These studies were both scientifically and practically approached to critically evaluate required outcomes. The process defined various criteria and controls to ensure the integration and consistency across the activities and projects as well as the achievement of goals and objectives. It took into account the main challenges and lessons learned from the earlier strategy work. The phases of strategy development included a series of key controls:

- Compliance of Emirates ID's mission with the vision and strategy of the federal government;

- Establishing Emirates ID's Vision based on its Mission;

- Identifying organizational values to support the achievement of Emirates ID's objectives;

- Determining strategic objectives and translating them into a Strategy Map;

- Defining strategic performance indicators for each strategic objective;

- Developing a number of initiatives and programs to meet the targeted objectives;

- Setting projects to implement the adopted initiatives and programs;

- Cascade objectives, targets, and projects across Emirates ID's organizational units; and

- Setting operational performance indicators for projects.

In the first stage of the strategy development process, Emirates ID examined and reviewed the components of the federal government's strategy stemming from the UAE Vision 2021 as a springboard for Emirates ID's strategic objectives. Emirates ID then identified the components and objectives relating to its own activities. Prior to defining the Mission, Vision, and Strategic Objectives, several assessments were made to identify where the previous strategy was succeeding and where there were any gaps. Emirates ID used PESTLE analysis to identify a series of political, economic, social, technical, legal, and environmental indicators and factors that could influence its strategy. These factors were then entered in the second half of the SWOT analysis (Strengths, Weaknesses, Opportunities and Threats).

The Federal Government Vision 2021 offered a clear overview of the external environment and how it would impact future development and initiatives. As a federal government entity, Emirates ID drew on this vision and the future opportunities and threats it encompasses. Emirates ID also sought to analyze the internal environment based on organizational best practices, "brainstorming," and "work groups" to identify strengths and weaknesses.

The experiences and practices of other countries were also used as international performance benchmarks. Emirates ID recognized the importance of drawing on the experiences and practices of other countries in implementing advanced identity systems in its search for cutting-edge inputs and approaches for building and formulating the outcomes targeted by its new strategy. Emirates ID was also determined to study the needs of all stakeholders and to involve them in the creation of its strategy.

Emirates ID also worked systematically to strike a balance between the needs and expectations of the various stakeholders in order to achieve positive outcomes for all stakeholders. All this constitutes imperative inputs for defining the general objectives of our strategy and to pinpoint the projects and initiatives that will enable the authority to achieve its strategic aspirations.

Emirates ID adopted a scientific approach in creating its vision and mission; expressing concisely and clearly its ambition, key message, and role in the community as well as its future aspirations. As indicated earlier, Emirates ID was keen to address all key stakeholders in the formulation of its vision and mission. The authority chose the wording carefully and weighed the possible implications of each term to make sure that the content was comprehensive, clear, and easy to understand by all concerned parties.

Emirates ID also recognized the centrality of corporate values and principles in building an optimal and effective work environment. These values and principles were management tools that created bonds between the organization and its staff. These values were seen as a driving force for the creation of a united corporate culture that channels human potential toward achieving the strategy's targeted outcomes.

As such, Emirates ID defined five corporate values as the foundation upon which its activities shall be built. These values were the moral code that guided performance and conduct as well as occupational and human relations within Emirates ID. The authority also worked toward instituting practical and realistic programs to consolidate these values in everyday activities. The different programs served as supportive management approaches (management through values and principles), which Emirates ID followed to reinforce the capacities of its staff in understanding, 
implementing, utilizing, and activating such values within the organizational system at Emirates ID.

Hence, a well-articulated strategy existed at Emirates ID and was always formulated based on a very structured framework. Figure 4 depicts the flow of major strategy development components that guided the formulation of the core strategy.

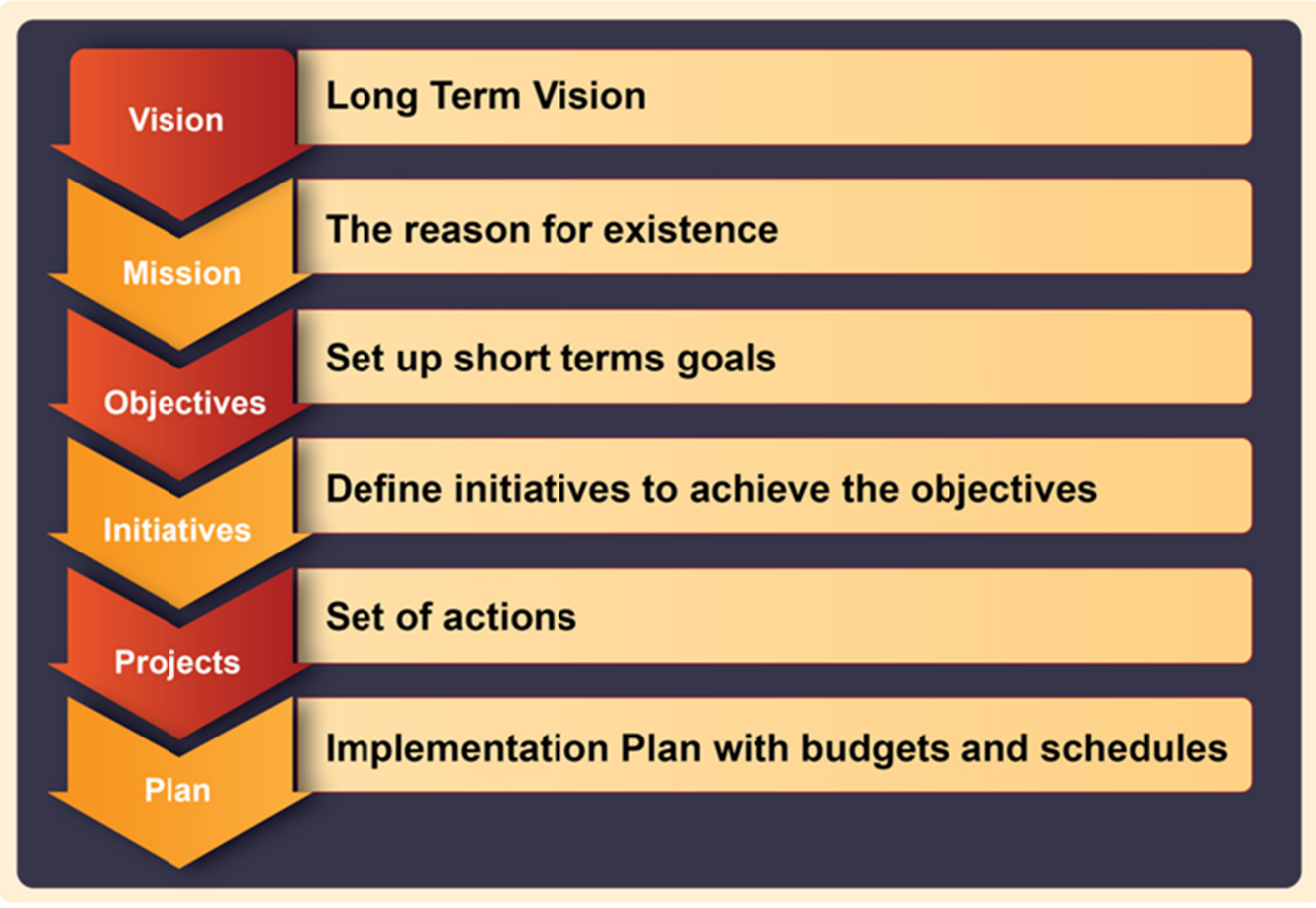

Figure 4. Interconnected Strategic Endeavour

The next step after developing the 2010-2013 strategy was to translate it into a Corporate Strategy Map supported by a Balanced Scorecard. The Balanced Scorecard facilitated the process of selecting the strategy's criteria and controls as well as the projects that would support the implementation of the strategy. The Balanced Scorecard system contained a Strategy Map and supported a scorecard of strategic measures, stretching targets, and strategic initiatives launched to close the gap between present and targeted performance (Kaplan and Norton, 1996). These were collocated according to outcomes and enablers (or non-financial) performance perspectives.

The argument was that the successful delivery of objectives in the enabler perspectives drives, through a causal relationship, success in the outcome perspectives. Based on the strengths, weaknesses, threats, opportunities, and key inputs identified through the above-mentioned analysis and studies, Emirates ID devised strategic objectives that have contributed to its strategic intents distributed across 3 Balanced Scorecard Perspectives:

- The Value Perspective described the ultimate outcome of the Strategic Plan. It represented Emirates ID's contribution to the development of the Country and the next step that must be accomplished toward the achievement of the authority's Vision.

- The Internal Processes Perspective described the Strategic Intents and Objectives that will drive the achievement of the Value Perspective. It represented the operational focus that must be followed during the next strategic cycle.

- The Enablers Perspective summarized the organizational needs that were required to the proper execution of the strategic Plan.

The definition of strategic objectives for each perspective was achieved through internal workshops in which senior managers, directors, and some middle-management staff participated. The objectives were discussed and their detailed descriptions were set according to structured scientific methods. The 2010-2013 Strategy Map was constructed based on 4 Strategic Intents, and each strategic objective was defined to serve specific Strategic Intents in a horizontal cause-and-effect relationship between the 3 perspectives. 


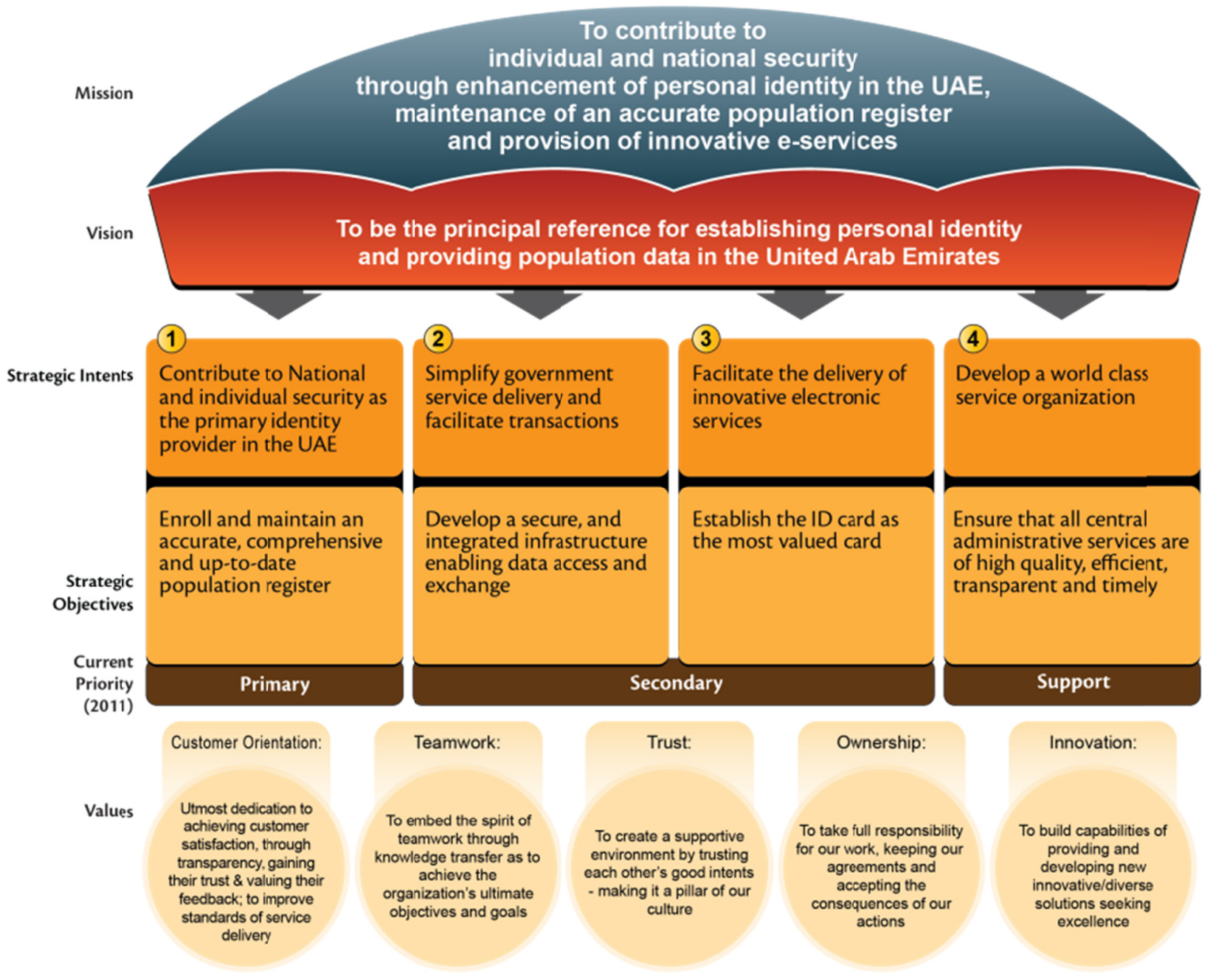

Figure 5. Emirates ID 2010-2013 Strategy Map

Management teams paid particular attention to the development of the strategy map that was later used as a communication medium across the organization. Figure 5 depicts the 2010-2013 strategy map. The simplicity of its content and illustration contributed to (1) faster circulation of the new strategy and acted as (2) a holistic visualized means for all departments and units of the overall organizational objectives and outcomes.

The strategy map was supported by a Balanced Scorecard with measures and targets across all perspectives. The scorecard framework was used as a management approach to monitor and measure performance and as a tool for internal and external communication. It included key performance indicators (KPIs) measuring the performance of each strategic objective of the organization across the value, the internal, and the enablers perspectives. It also included ambitious targets for each KPI for the years 2010-2013 as well as key initiatives that Emirates ID had to undertake in order to meet those targets.

Each measure was assigned to a KPI owner responsible for achieving and reporting on its performance. KPI owners, descriptions, calculation methods, analysis, and recommendations were provided through KPI cards. Objective status was also provided through Objective status cards monitored by the Objective Owners. Emirates ID Authority not only reported on its performance internally to the leadership team, but also to the Prime Minister's Office to ensure alignment to the federal strategy.

\subsubsection{Human Resources Development}

The other major element of the inner circle focused on monitoring the contribution of Emirates ID's employees-its most important intangible asset - to the efficacy of the organization: Human Resources. An international framework was used to train and develop staff and improve their performance. HR Development was fully aligned with the core 
strategy and adopted the framework depicted in Figure 6. This offered a robust platform for continuous improvement of employee satisfaction, retention, motivation, and development. The framework further served to guide the correlation between employees' skills and capabilities and the organization's overall strategy.

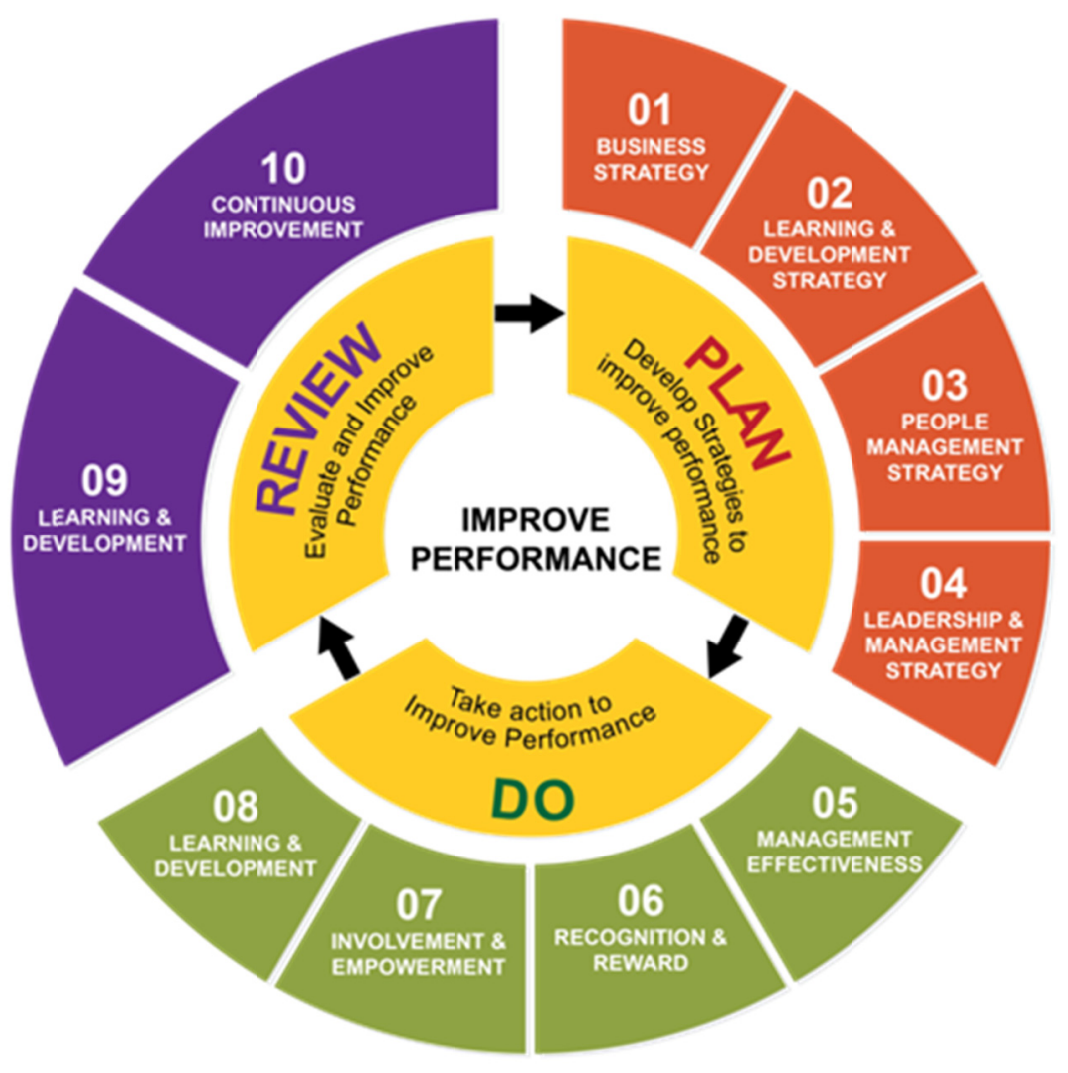

Figure 6. Investors in People Framework

\subsubsection{Business Process Reengineering}

Business process is the inherent and defining element of any organization. It is the base for any activities and its correct and optimal implementation has a direct influence on the success or failure of any organization or strategic endeavor. Process management and process optimization thus served as a corner stone for organizational efficiency at Emirates ID. The 2007-2010 strategy at Emirates ID was designed with a transformation orientation; hence it involved radical redesign of core business processes in order to meet the expected results regarding the quality of the service delivery and the experience provided to customers.

New processes had to be embedded in the organizational structure, which called for periodic reprioritization of initiatives. Business processes were re-designed iteratively to add value in terms of increased effectiveness (value to customers) and increased efficiency (lower costs). Process reengineering contributed fundamentally toward:

- Costs and cycle time reduction: reduced costs and cycle times by eliminating unproductive activities and improving the employees' throughput; and

- Improve quality: reduced the fragmentation of work and established clear ownership of processes. Employees were assigned clear responsibilities for their outputs through balance scorecards that allowed them to measure their performance on a daily basis.

Figure 7 depicts some major characteristics of the BPR initiative at Emirates ID.

Emirates ID also used benchmarking to measure and improve performance of our key processes. Process benchmarking was used to evaluate various aspects of identified processes in relation to best practices in operations and customer service. Benchmarking was considered as a powerful management tool to avoid "paradigm blindness" in the mode of thinking, i.e. to avoid thinking like "the way we do it is the best because this is the way we've always 
done it."

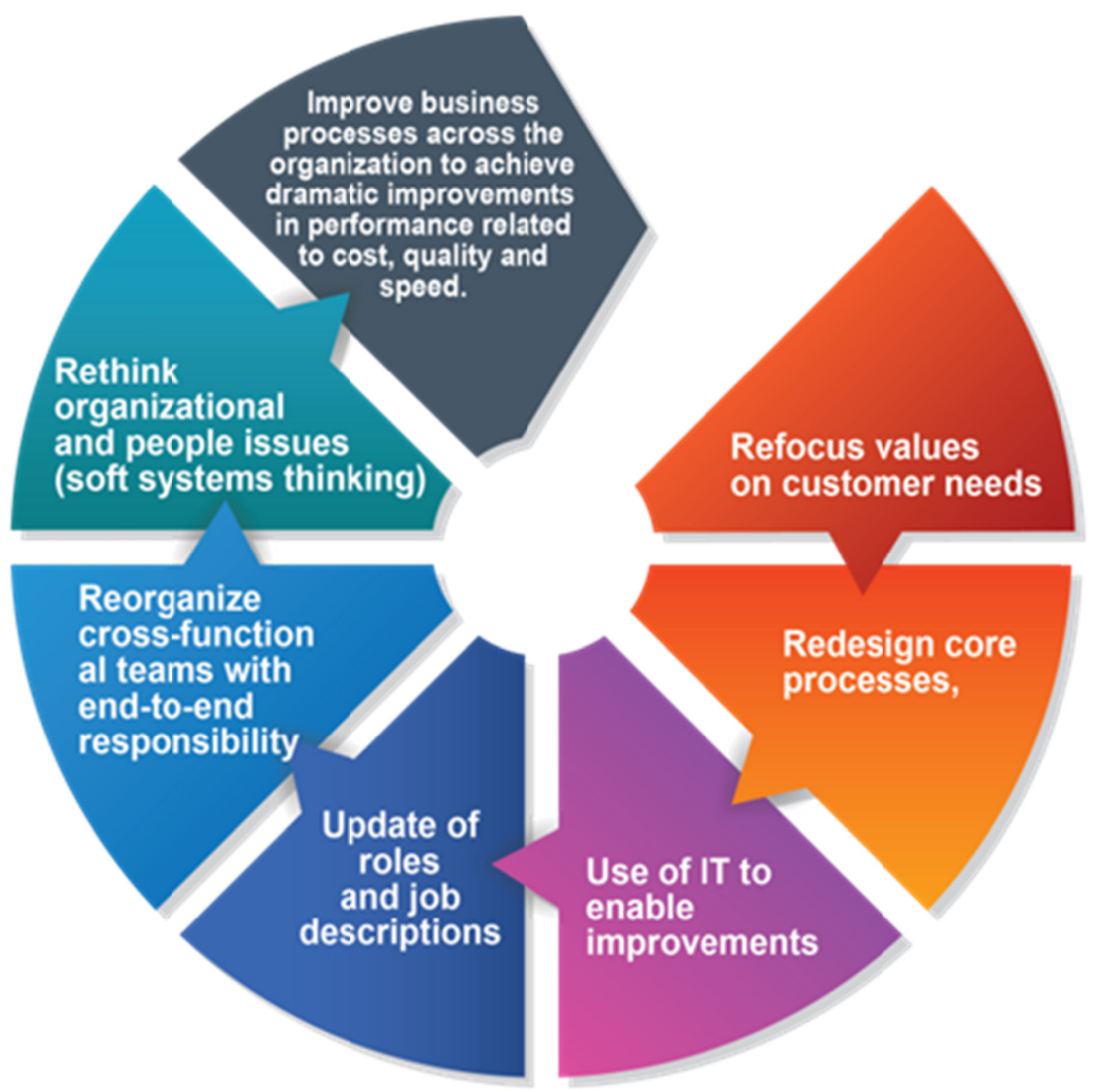

Figure 7. Key BPR Drivers at Emirates ID

Benchmarking opened the organization to new methods, ideas, and tools to improve its process effectiveness. It helped foster a mental shift as it cracked through resistance to change by demonstrating other methods of solving problems and organizational excellence. Emirates ID also opted to outsource limited functions and processes through a public-private-partnership model. These involved Build-Operate-Transfer (BOT) strategies as a form of project financing that were transferred to the organization at the end of the concession period. These outsourcing contracts lasted from two to four years. The next two sections will further outline the impact of process reengineering and outsourcing on the financial performance of the organization.

\subsubsection{Customer Centricity}

Emirates ID adopted a value system that placed increased emphasis on customer needs. (See also section 4.2.5). Emirates ID's core strategy was customer-driven, and the entire business operating model was reoriented around customers and enhanced customer experience. Figure 8 depicts a high-level model that was followed at Emirates ID.

Extensive efforts were put to create a customer-centric culture. Redesigning the organization to put customers at the center of the business model and business decisions was perplexing, especially when talking about a government agency that is routine in its character and change-resistant by nature. Figure 9 depicts the holistic approach of elements used to reshape organizational culture at Emirates ID with the goal of improving customer experience.

Emirates ID had to go beyond strategy, technology, policy, and process elements to focus on delivering its customer-service vision and nurture the behavior it needed to model and adopt. Initiatives included organizational change management, leadership and mind-set solutions, customer-focused development programs, and reward systems - all driven by strong internal communications. 


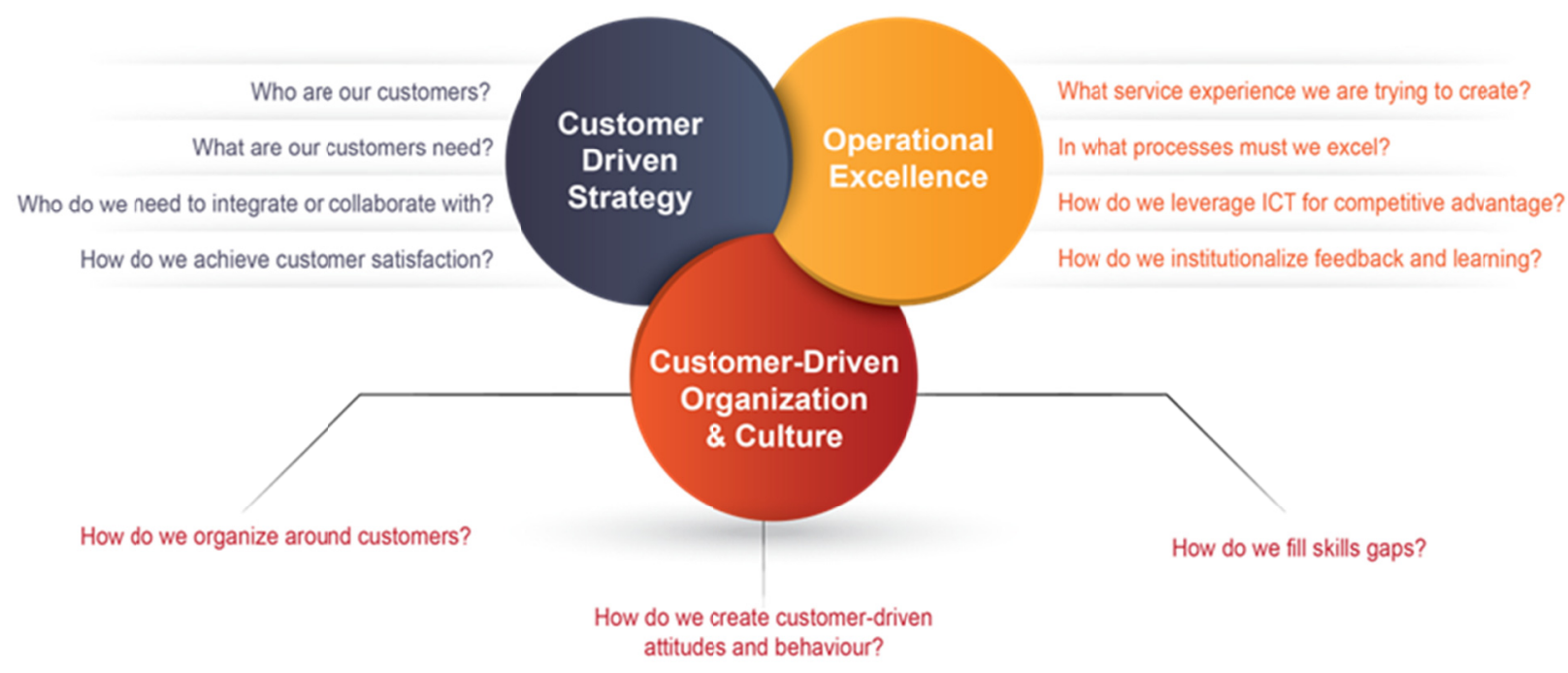

Figure 8. Customer-Driven Business Model Source (Gavan, 2012)

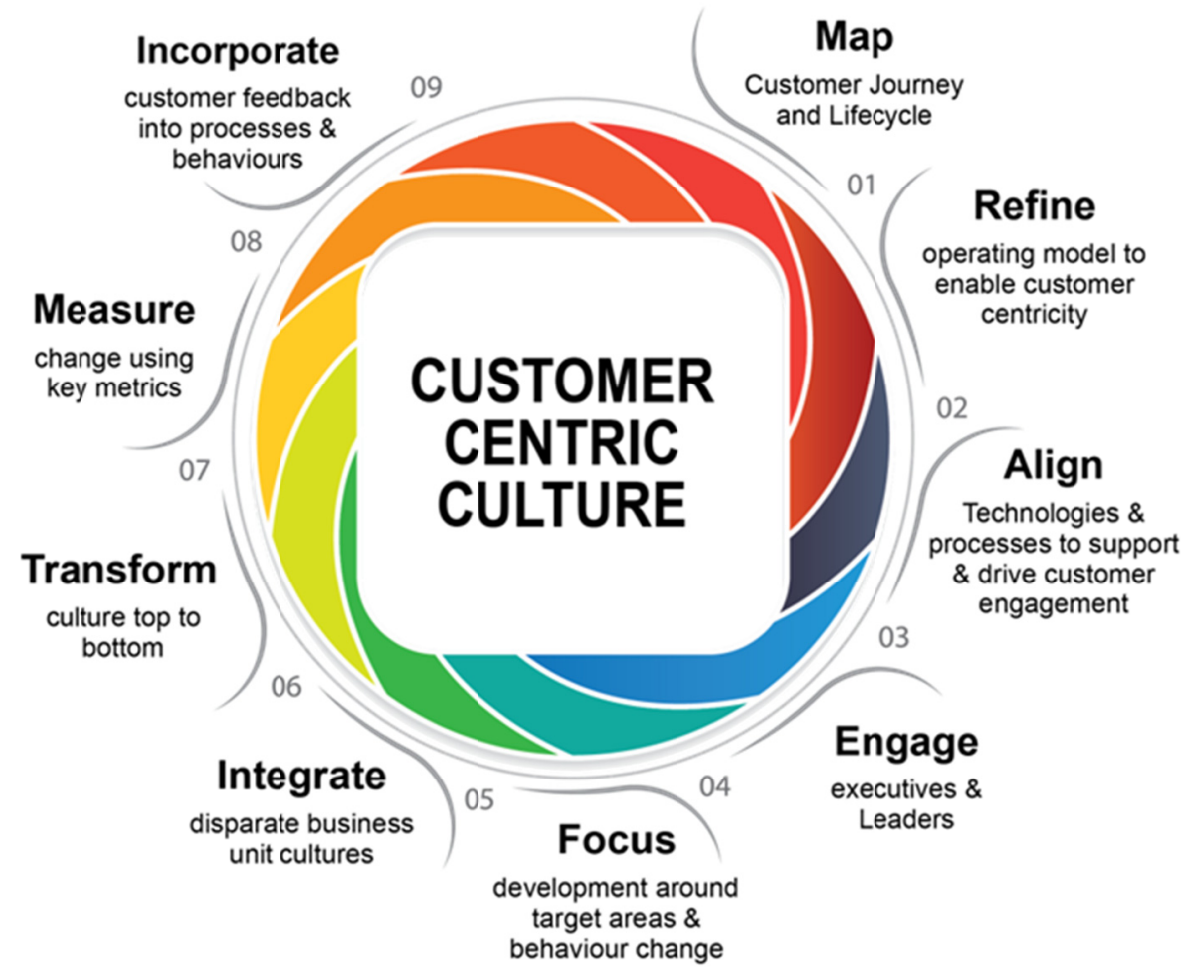

Figure 9. Customer-Centric Culture

Source: (Cascio et al., 1997)

The approach to customer service at Emirates ID was dynamic and ROI driven, and it was often challenging and uncomfortable. Customer expectations were envisaged to be met at every interaction with a defined consistency of service delivery and experience. Key results from such orientation included customer-service targets to reach the highest in the country. See also KPI's in table 2 of section 5 below. The continuous upward trend clearly indicated sustainable performance. Emirates ID was selected as the best federal entity in service provisioning for two award cycles, 2012 and 2014, in the UAE Government Excellence Program. 


\subsubsection{Financial Performance}

The fifth element in the inner circle is financial performance. It is an imperative that serves as a constraint to drive performance optimization. Emirates ID depended on annual government financial support. However, the allocated budgets were not sufficient to allow it to fully embark on planned projects defined in the new strategy, as almost $70 \%$ of the budget was spent on salaries and operational expenses. The primary revenues were envisaged to come from the enrolment fees, i.e., the fees collected from applicants seeking ID card issuance. However, with weak operational performance in the initial years, the revenue was low, which in turn constrained implementation of new initiatives. With a fixed annual grant as government assistance, the organization was under pressure to find alternative options to finance its new initiatives.

Most organizations in such situations tend to reduce their human resources through layoffs or downsizing to improve performance and increase competitiveness (Cascio et al., 1997; Thornhill and Saunders, 1998). This is based on the assumption that reduction of costs related to HR will have a positive impact on the financial performance of the organization. But this was not the case at Emirates ID, even though the organization had a poor financial performance until the end of 2009. The management team looked at other measures such as opportunities to maximize revenue, operations optimization, and customer demand and developed an action plan to provide a better balance between supply and demand.

The business process re-engineering initiative played a key role in making the paradigm shift. The organization was able to make savings that exceeded US $\$ 50$ million annually. The outsourcing approach also provided the organization with more finance options. Outsourcing was considered as a pressure valve to reduce fixed costs. See also Figure 10.

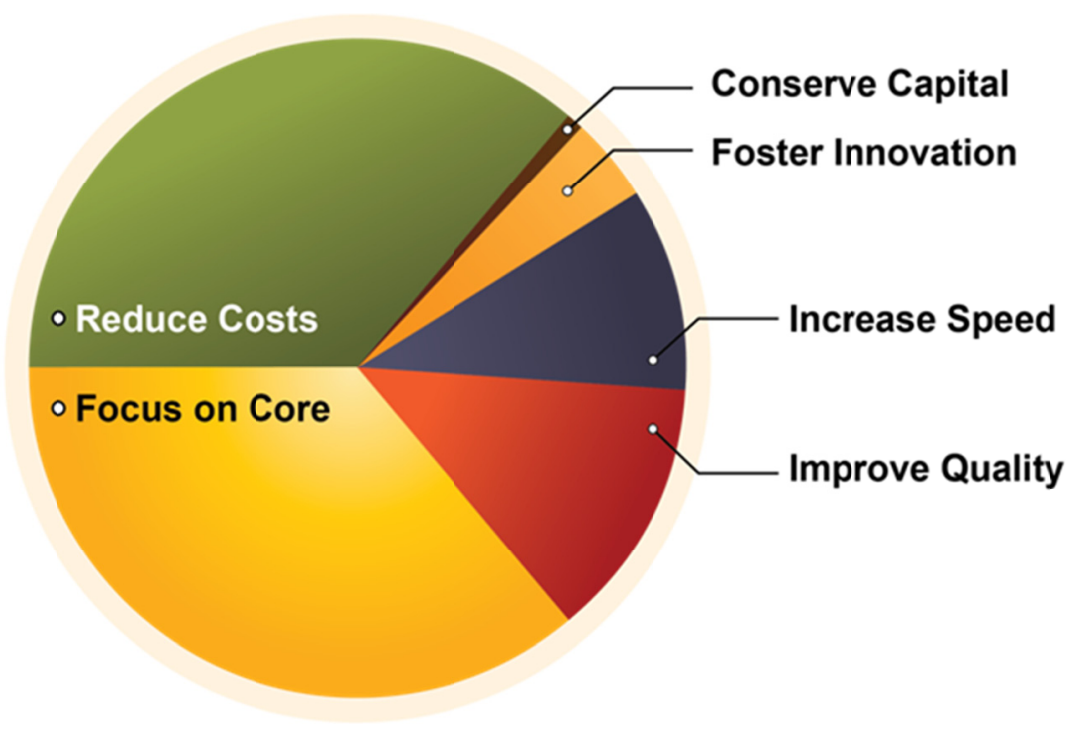

Figure 10. Perceived Benefits of Outsourcing at Emirates ID

A common thread running through all these approaches was the objective of cost-optimization and the need to demonstrate a robust return on investment for every unit spent. All in all, as the organization focused all its efforts in fulfilling its core functions (enrollment of the population), the financial performance was tightly coupled with it. Although the organization operating expenses went up by almost 4 times with the new strategy, Emirates ID stood to make surpluses year after year due to its outstanding performance in its core business (population enrollment). Another result of this "unconventional effort" to improve financial performance, workforce recruitment went up by more than 400\%; from 250 employees in 2009, to more than 1,000 in 2014. 


\subsection{Tier 2: Setting the Tempo Symphony at Organizational level}

The second tier of the framework represented foundational business practices that needed to be developed in order to sustain organizational excellence and competitive performance. See Figure 11. Each of these functions is discussed next.

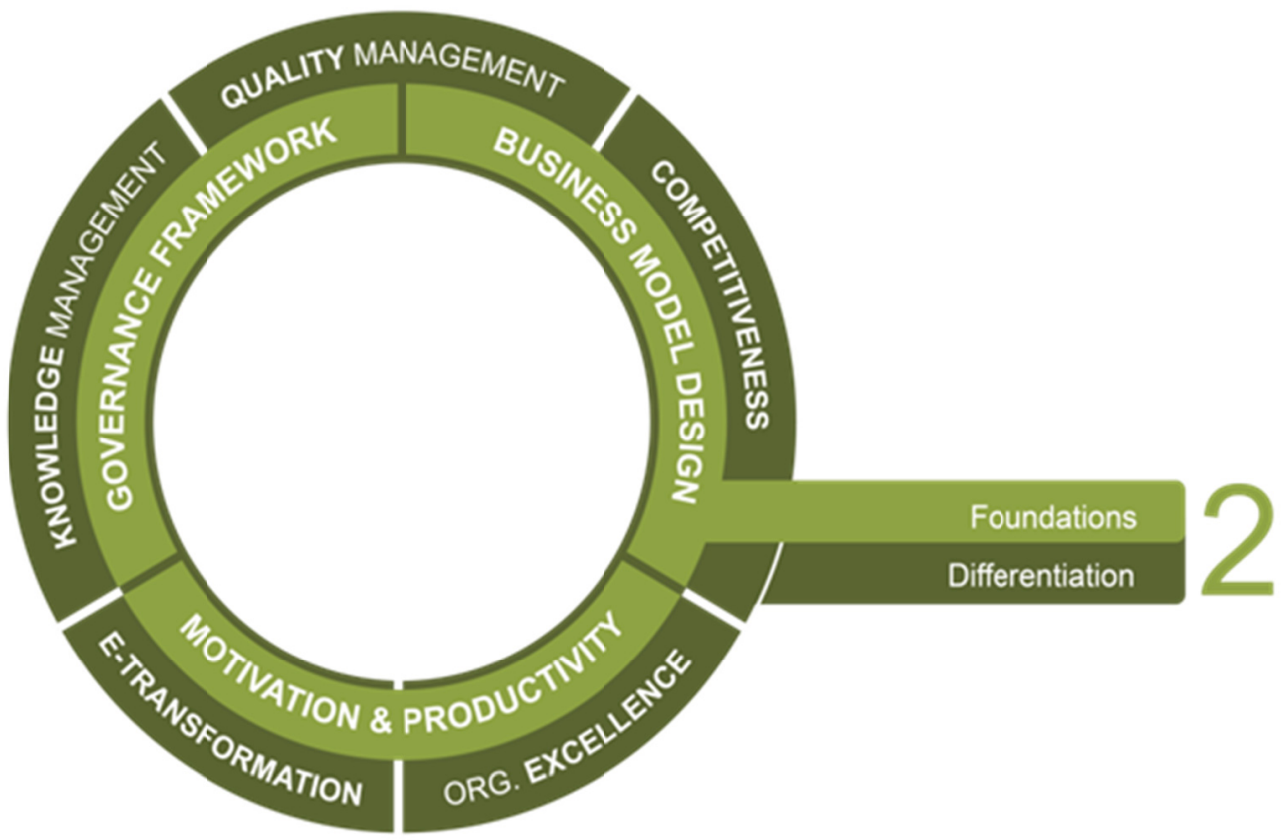

Figure 11. Organizational Excellence Foundations and Differentiation

\subsubsection{Business Model Design}

The new transformation strategy required careful and advanced preparation and planning to address programmatic needs, support services to meet organizational goals, and effective workforce planning and communication methods to be put in place. As a pre-requisite to proper workforce planning, Emirates ID reviewed its business model and the organization structure that supported it three times to coincide with the strategy cycles-beginning of the strategy, midway after two years, and toward the end - with the announcement of the new strategy. Realizing that change management is critical to the implementation of the strategy and organizational development, a phase-based operating model was developed, enabling the desired transition. This is depicted in Figure 12.

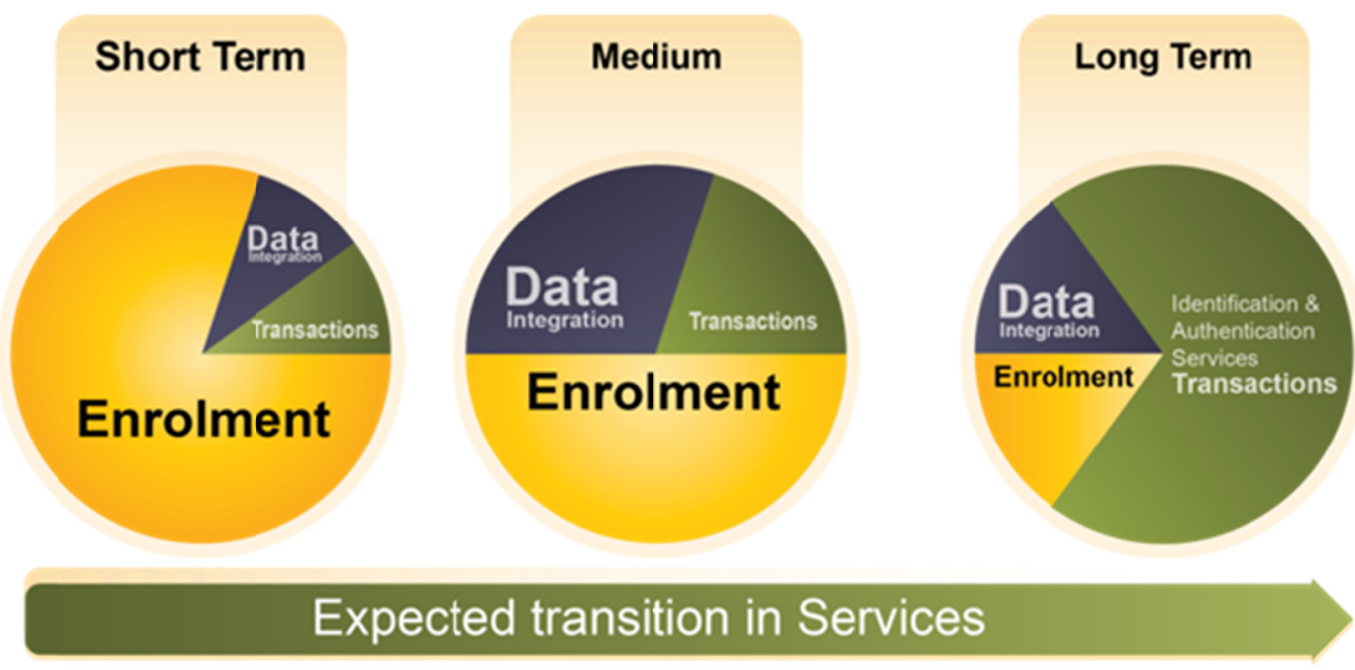

Figure 12. Emirates ID’s Business Operating Model 
The goal was always to identify the best people to meet organizational objectives and challenges at each stage of the strategic plan and to progressively incorporate strategic capabilities before they become critical. In fact, a continuous challenge was the optimization of human-resource utilization and reallocation of existing talent based on the shifts on Emirates ID's strategic focus, while recognizing the limitations of mind-set boundaries in its staff both at management and operational levels. See also Figure 13.

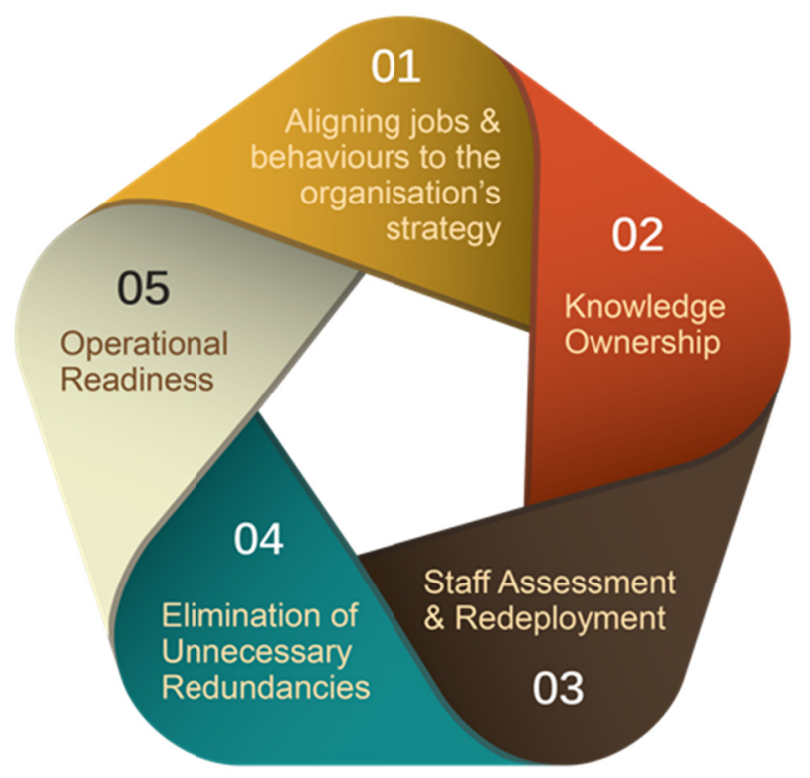

Figure 13. Primary Restructuring Objectives

Business model redesign and organizational restructuring also required re-articulation of the roles and responsibilities to ensure unambiguous and fast decision-making; the development of values and norms for cultural alignment with strategy; design of rewards and metrics, and the identification of key capabilities (skills and knowledge) required to execute strategy. See Figure 14.

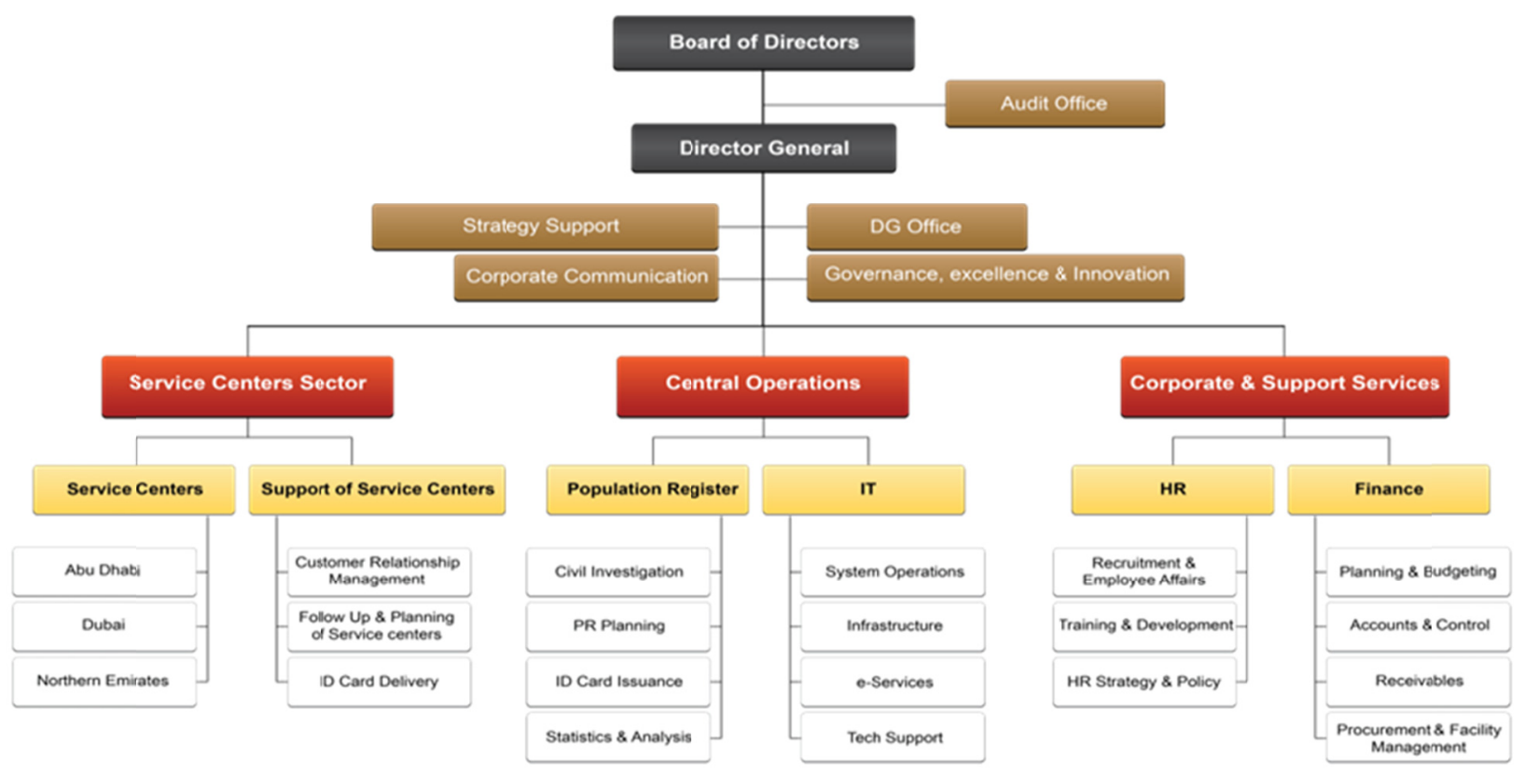

Figure 14. New Organization Structure 
Preliminary transition plans were shaped to develop design steps within the context of a change management agenda. (See also section 4.3.1). Each restructuring activity focused on reducing organizational layers and eliminating unproductive activities in two key areas. First, it redesigned functional organizations into cross-functional teams. Second, it used technology to improve data dissemination and decision-making.

\subsubsection{Governance Framework}

A robust governance framework was essential for the organization to operate effectively. Governance processes were put in place to ensure acceptable performance. Figure 15 depicts a general view of the corporate governance framework developed. It was used as a tool to show how the organization should use formal governance arrangements to contribute to its overall performance and the delivery of services and programs. Primarily, it encompassed conformance requirements to ensure that it meets the requirements of the law, regulations, published standards, and public expectation of service quality.

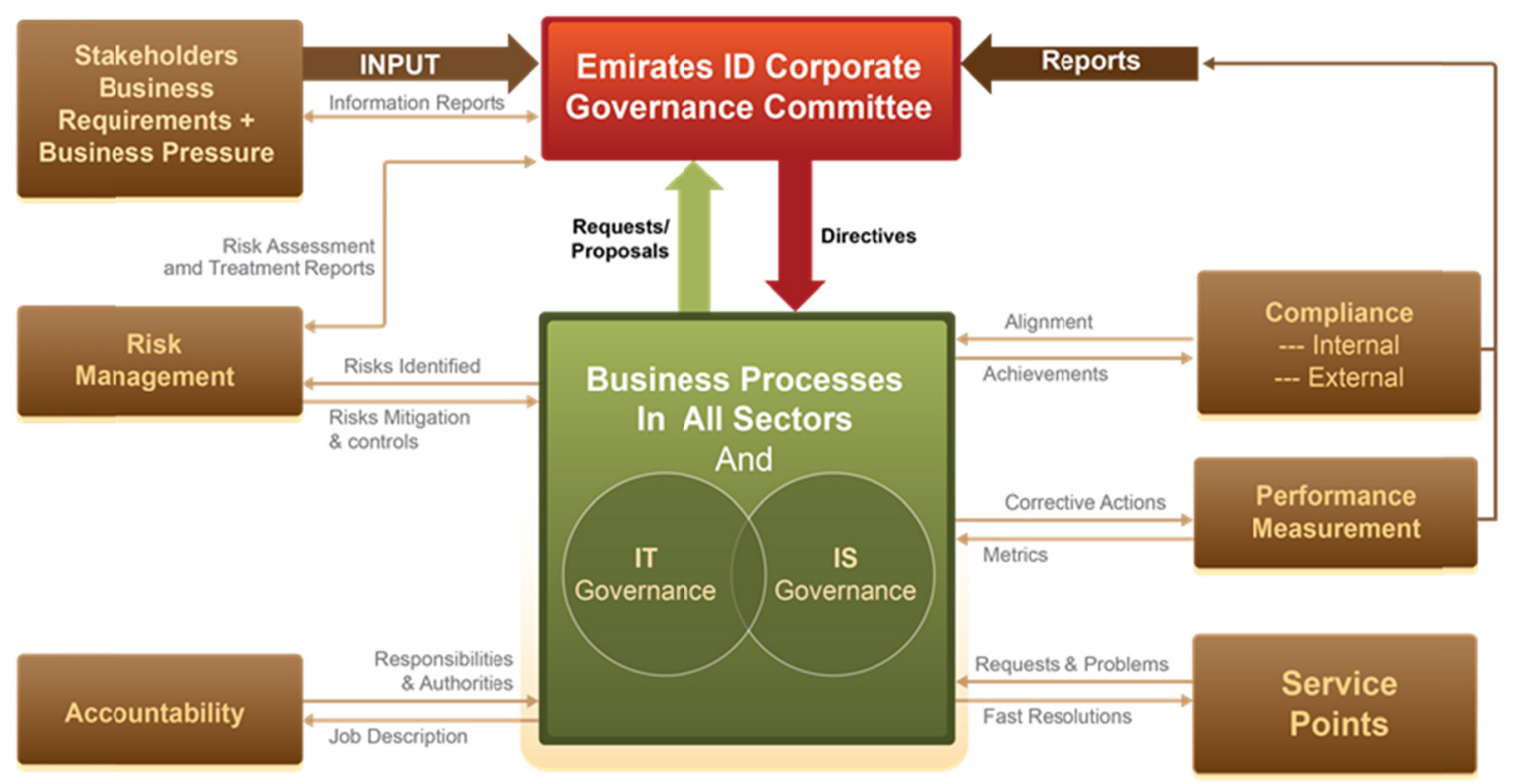

Figure 15. Emirates ID Governance Framework

The Corporate Governance Framework outlined the principles, elements, and mechanisms used within the organization to support effective corporate governance through strong leadership, responsibility, and ethical decision-making, management accountability, and performance management. Emirates ID created and frequently updated different policies and procedures (business methodologies) that were aligned with the EFQM model standard. These documents served as backbone for the overall governance framework. (See also section 4.2.4).

\subsubsection{Motivation \& Productivity}

Motivation is the most critical factor in productivity. There were several approaches that Emirates ID has put in place to motivate its employees and drive behavior and productivity. For example, it used different incentive schemes to motivate employees meet short-term and mid-term performance goals. To achieve long-term goals, Emirates ID implemented programs to encourage competition between employees and between the departments to meet strategic objectives. Top performers were recognized on a monthly and yearly basis. Motivation was seen more a like a muscle.

Emirates ID needed to practice strengthening it through a regular routine (see for example: Cohen, 2013). Management focused on building and providing the conditions under which employees would be motivated and worked to satisfy the needs that would contribute to performance. Figure 16 depicts examples of how different needs were satisfied using Maslow's hierarchy of needs. 
Challenging \& performance driven working environment | Comprehensive training \& personal development plans | multiple self-learning resources | support continuous learning | Financial support \& flexible work hours for education pursuit | Instituting Organizational Learning center for individual \& group learning | Promotion \& career progression program | Promote R\&D, creativity \& innovation culture | Skill, competencies \& talent development

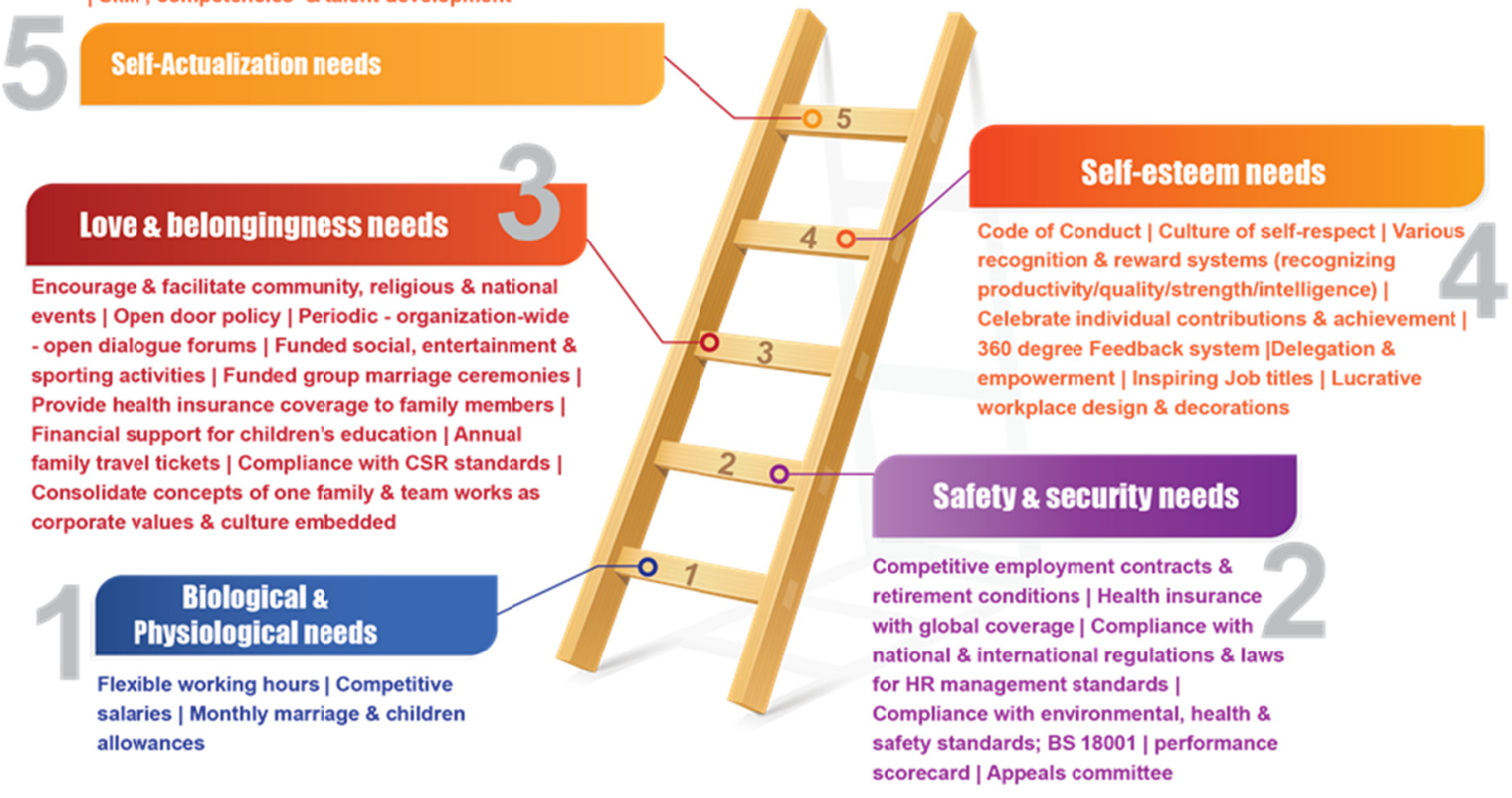

Figure 16. Maslow's Hierarchy of Needs Source: (Maslow, 1943)

4.2.4 Quality Management
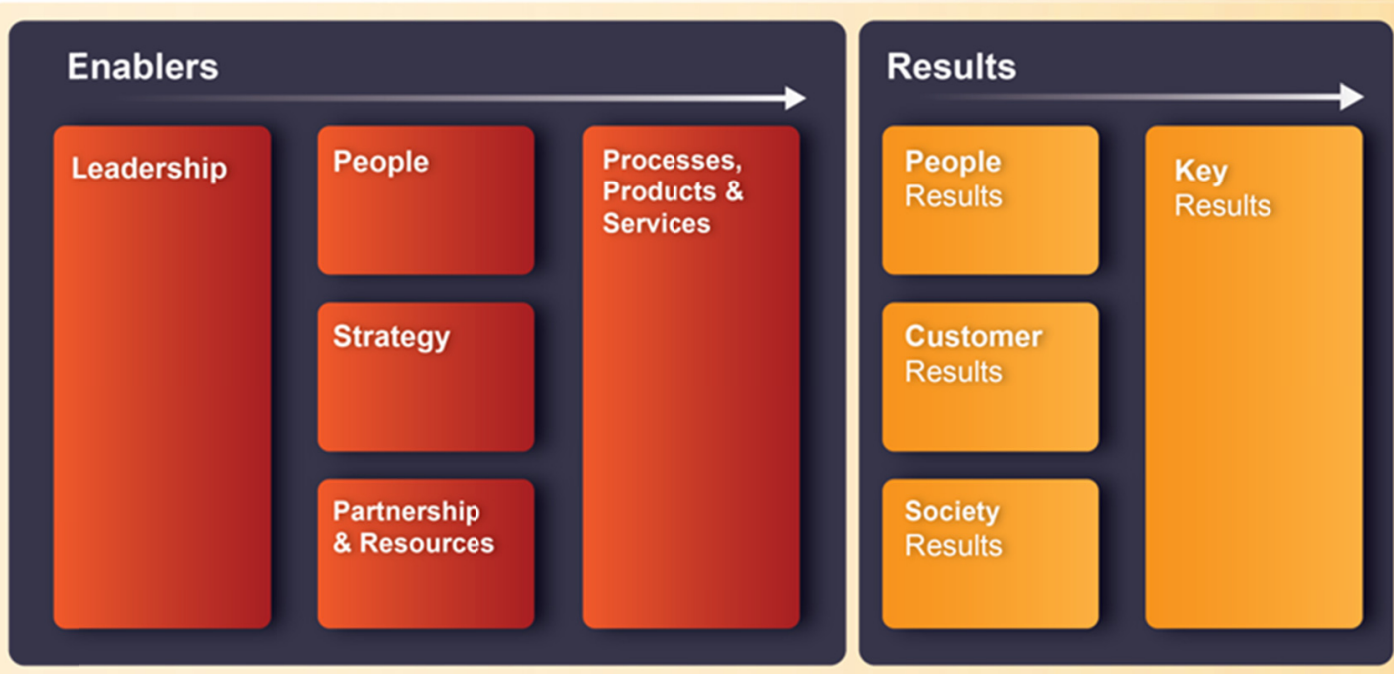

Learning, Creativity and Innovation

Figure 17. EFQM Excellence Model

Operational practices at Emirates ID by and large were driven by total quality management-TQM principles. It thus 
considered the European Framework for Quality Management (EFQM) model as an operational framework for TQM (Note 2). The EFQM Excellence Model, which relies on the principle of continuous improvement as an overall approach, is made up of nine elements grouped under five enablers and four result criteria. See also Figure 17. The enablers represent the way the organization operates, and the results concentrate on achievements expected by organizational stakeholders (EFQM, 2015).

The EFQM excellence model helped the organization measure delivery against strategic goals defined at the Corporate Balanced Scorecard, brought consistency to management style, and derived innovation. It stood as a tool to guide the development of organizational excellence. Different methodological systems and procedures were developed to address EFQM dimensions that were grouped in six primary clusters. Figure 18 provides an overview of them.

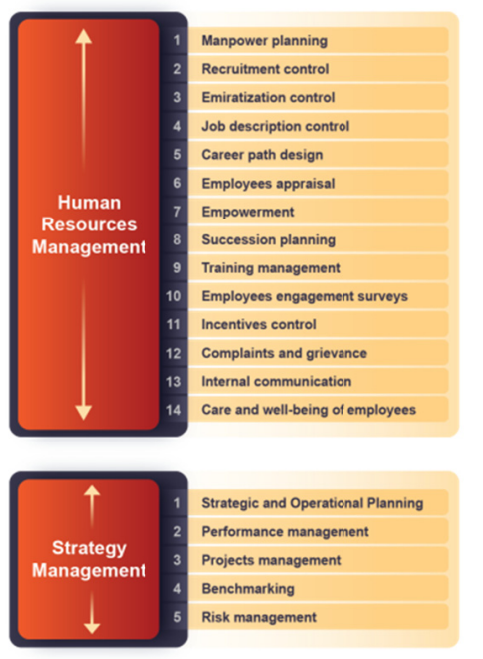

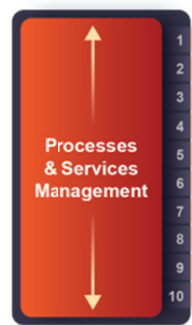
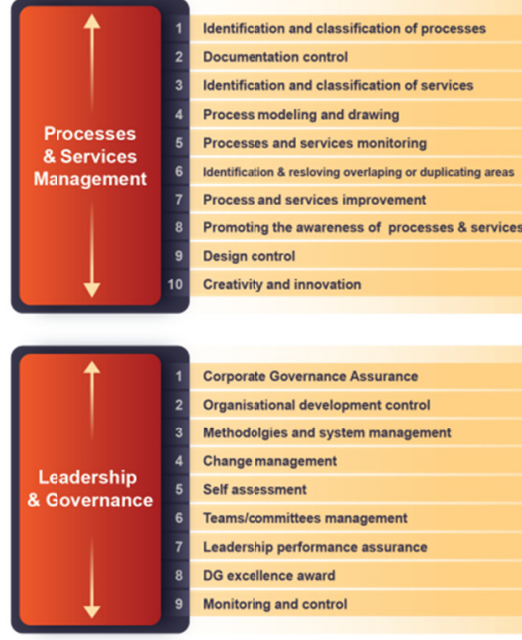

Corporate Governance Assurance Organisstional development control Methodolgies and system management Change management Self assessment Teams/committees management Leadersnip performance assurance DG excellence award Monitoring and control

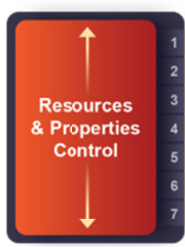

Budgeting control Revenues Development \& rationalize expenditures Financial impact assessment Financial reporting Asset manegement Technological assets development and control Knowledge management

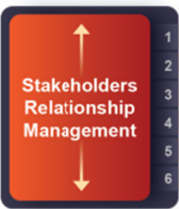
Partnership relations management Suppliers relations management Customers relations management Social resposibility Governmental communication managemert Suggestions and complaints handling

Figure 18. Policies and Procedures Developed in Accordance to the EFQM Excellence Model

Besides, Emirates ID used international standards to ensure compliance with international codes of practice for various administrative and technical matters. ISO 9001, ISO 26000, ISO14000, ISO 16001, ITIL, TICSI, and IIP are all examples of some of the adopted standards. These standards acted as extremely useful tools and guidelines and helped the organization tackle some of the most demanding challenges related to operations efficiency, productivity, and customer service. The primary benefits can be summarized thusly:

- Process optimization and cost savings: helped optimize operations and improve bottom-line results;

- Enhanced customer experience: improved quality, enhanced customer satisfaction, and more efficient and effective service delivery;

- Effective ICT usage: implement and manage ICT systems according to industry compliant and best practices;

- Organizational excellence: increased productivity and competitiveness;

- Environmental benefits: reduction of negative impacts on the environment; and

- Social responsibility: provided guidance on how to operate in a socially responsible way that contributes to the health and welfare of society.

\subsubsection{Competitiveness}

Around the globe, governments are challenged with issues that range from meeting the demands of citizens to enhancing the competitiveness of their jurisdictions while managing resources efficiently (Zibret et al., 2009). Emirates ID paid particular attention to develop and sustain competitiveness related to provisioning of services, utilization of resources, and knowledge management. Emirates ID used the value chain concept as a tool to analyze internal organizational activities and instill competitiveness (Note 3). See also Figure 19. 


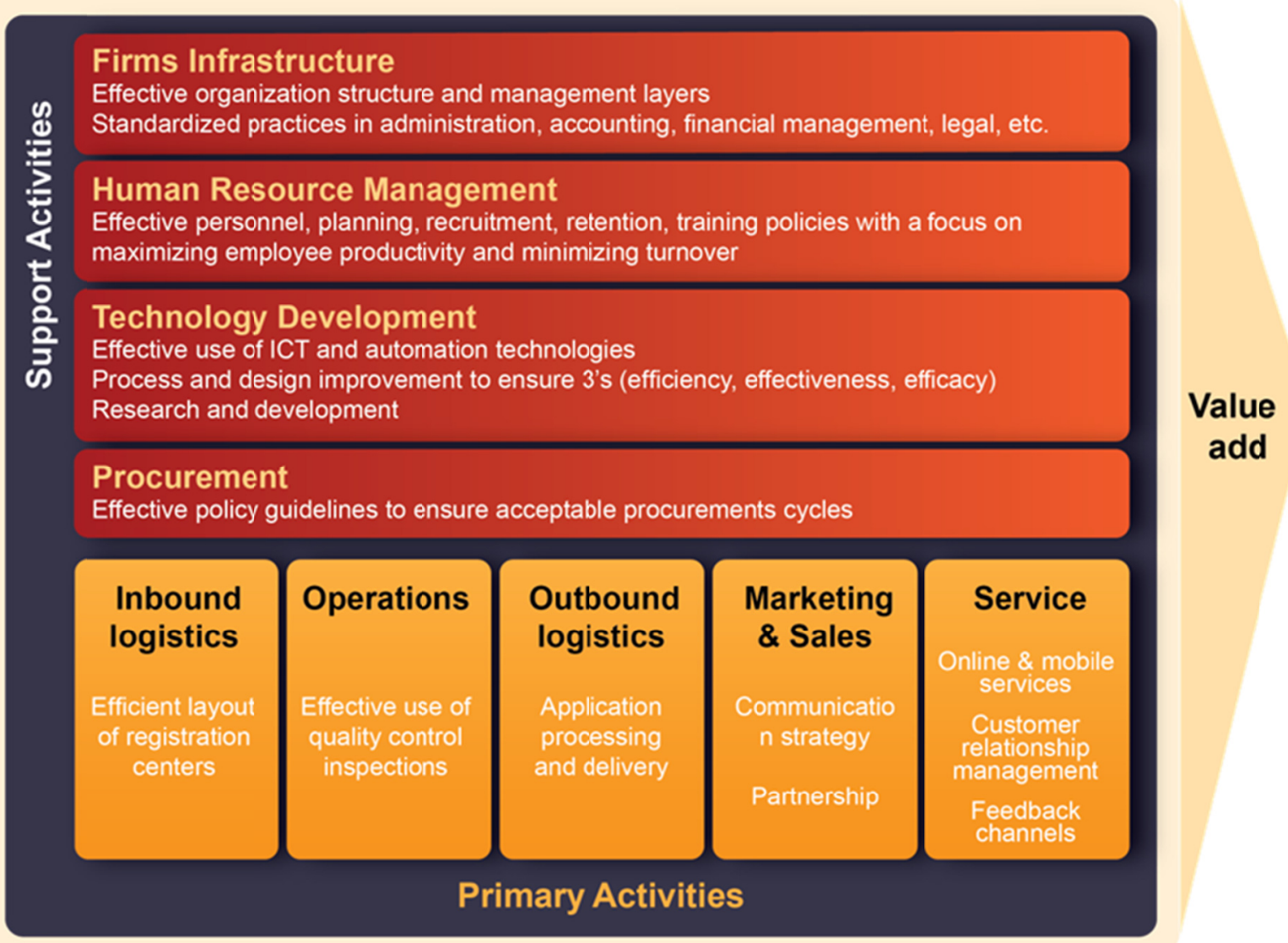

Figure 19. Value Chain Analysis at Emirates ID

Understanding how Emirates ID could create value and looking for ways to add more value were critical elements in developing a competitiveness-enhancement culture. Emirates ID began with a generic value chain and gradually matured with relevant value-creating activities. A linkage existed when the performance of an activity affected that of another. Understanding the linkages between activities led to more optimal make-or-break strategic decisions and served as a key input to the business model redesign and organization restructuring described previously. As such, competitive advantage was obtained by optimizing and coordinating linked activities. Value chain development at Emirates ID followed three sequential steps:

(1) Break down the organization into key activities under each of the major headings in the model;

(2) Evaluate the differentiation initiatives for improving customer value and meet the strategic targets related to the Customer perspective of the Balanced Scorecard, as well as identify current activities where the organization appears to be at a competitive disadvantage; and

(3) Determine and prioritize action plans built around focusing on activities where competitive advantage can be sustained and/or created.

Although primary activities added value directly to the core functions of the organization, they were not more important than support activities. Competitive advantage was mainly derived from technological improvements or innovations in business models or processes. Therefore, such support activities as 'information systems,' 'R\&D,' and 'general management' were usually the most important source of competitive advantage.

Another approach followed by Emirates ID to instill competitiveness into our organizational culture was through the introduction of a structured process for the adoption of innovation. In fact, the innovative mindset in the organization is an important factor that distinguished the organization and enhanced its competitiveness. According to a study conducted in the first quarter of 2014 to assess Emirates ID's innovation management performance and capability — based on the IMP3 rove Assessment-it was shown that the organization was above average for the 
benchmarked class and close to the rate of global growth champions (Note 4). This evaluation assessed the five dimensions of innovation: strategy, organization and culture, life cycle processes, enabling factors, and results. Figure 20 depicts the IMP'rove framework and an overview of Emirates ID's score.

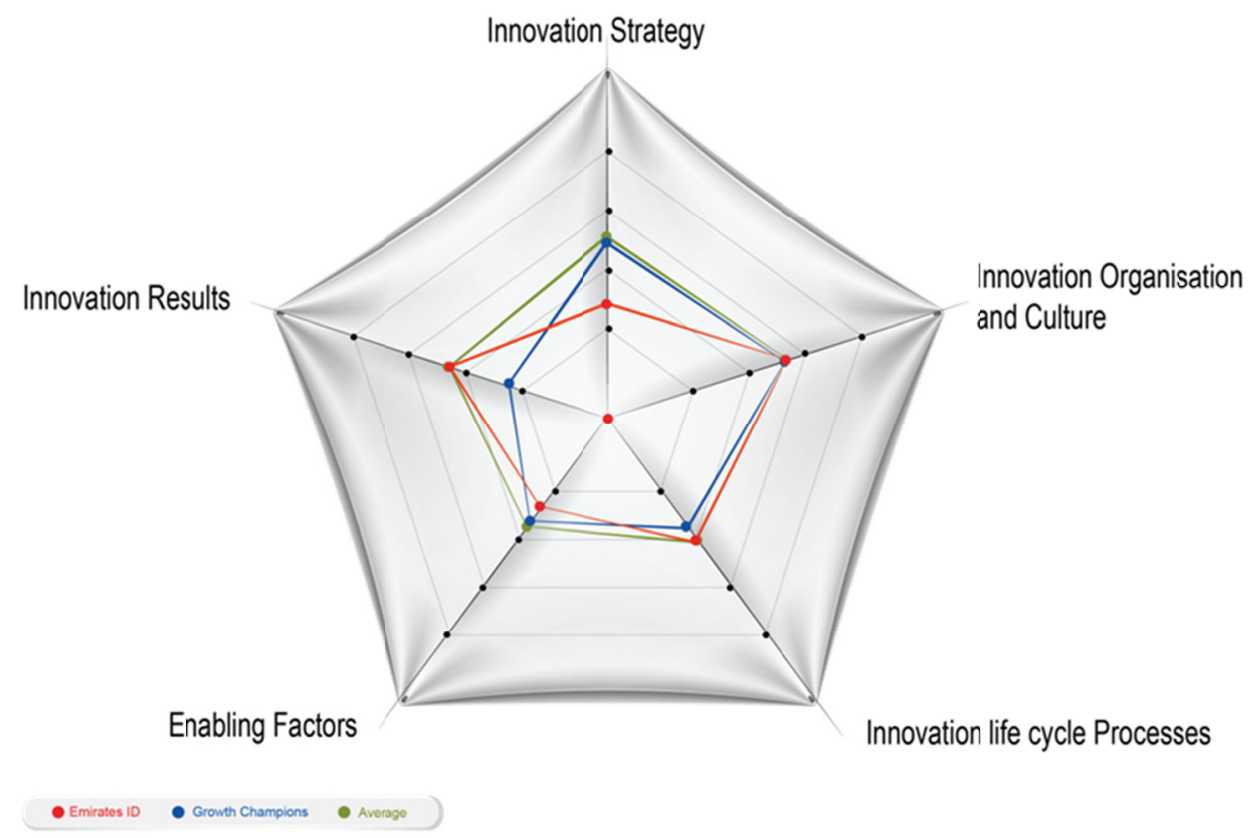

Figure 20. Emirates ID's Innovation Measurement According to IMP ${ }^{3}$ rove Framework Assessment in 2013

\subsubsection{Knowledge Management and Organizational Learning}

Emirates ID paid significant attention to knowledge management and organizational learning since 2009 (Al-Khouri, 2014). Knowledge management and organizational learning activities focused on supporting the sharing of best practices and lessons learned across the different units and organizational layers, constantly stimulating continuous improvement of the organization. See also Figure 21.

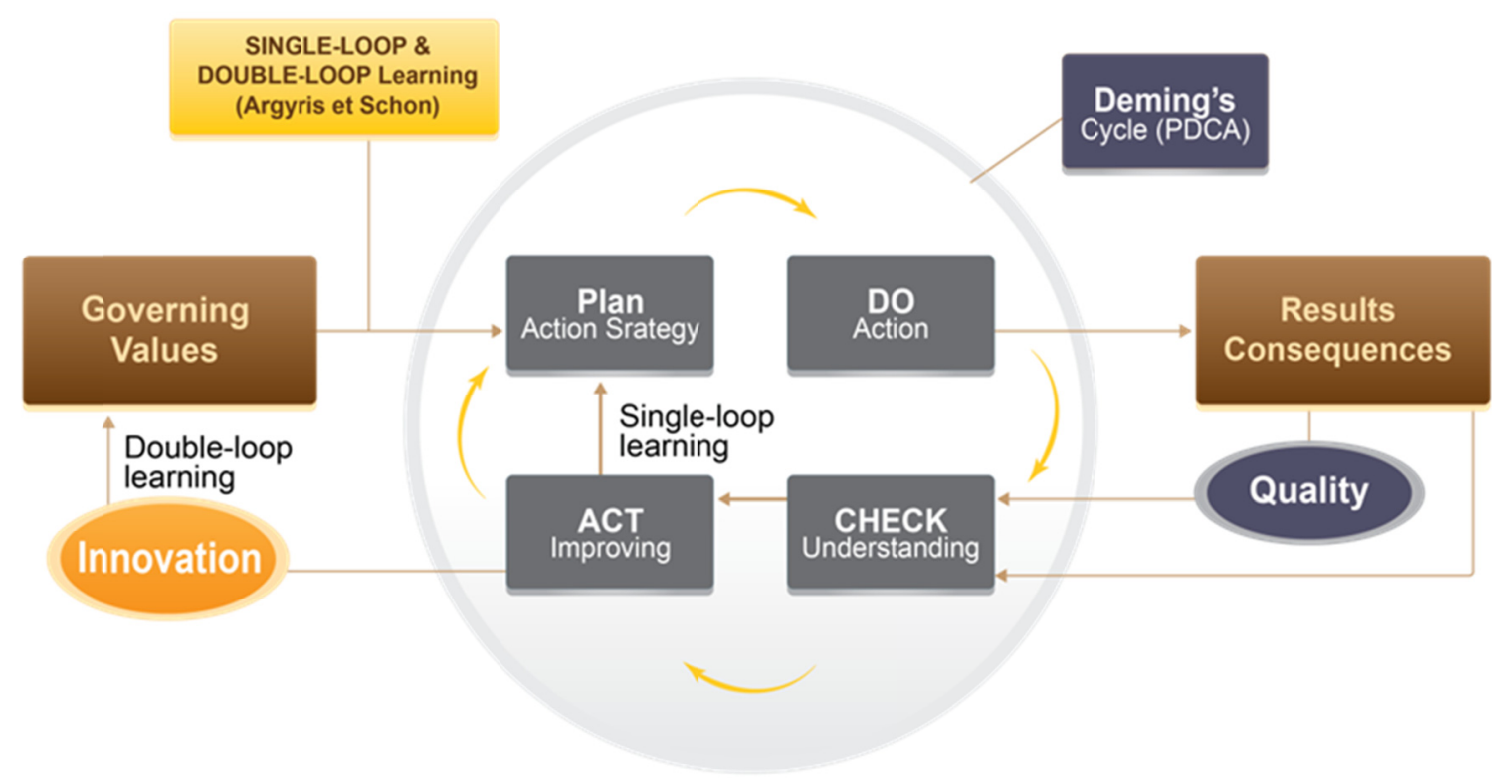

Figure 21. Continuous Development and Organizational Learning Source: (Grundstein, 2007) 
Through knowledge management, Emirates ID was able to consciously and comprehensively gather, organize, share, and analyze its knowledge in terms of resources, documents, and people skills. An automated system was developed to provide management teams the ability to organize and locate relevant content and the expertise required to address specific business tasks and projects. Emirates ID, as a learning organization, worked to integrate individual learning into organizational learning. Attention to individual learning supported learning at individual, group, departmental, and organizational levels in terms of problem-solving skills and implementation of solutions. There was a clear shift in employees' mindset at least in differentiating between terms such learning disability, single-loop, and double-loop learning. Organizational learning contributed to the advancement of creativity and innovation in the organization and supported the strategic orientation of the organization to develop and sustain corporate excellence.

\subsubsection{E-Transformation}

As an organization providing digital identities, Emirates ID is a technology-driven entity. The organization utilized IT for an effective internal e-transformation leading to mature e-services for its customers. Business-process automation not only resulted in huge contribution to the implementation of Green Policies, but also provided an impetus to service delivery mechanisms. Many services - both internal and external-were transformed from physical over-the-counter services to self-services available across a host of electronic channels that included the Web, Self-Service Kiosks, and Mobile Phones. The E-transformation was again a carefully calibrated process derived from Emirates ID's strategic guidelines, which resulted in higher customer satisfaction. The path for transformation was from a system-based and technical-driven service organization to a capability-driven and business-focused organization. See Figure 22.

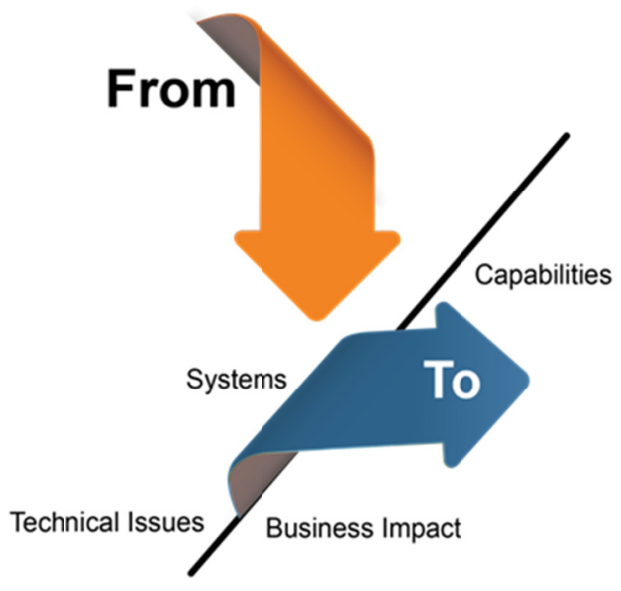

Figure 22. e-Transformation Path

The E-transformation thus resulted in enhanced employee and customer experience while interacting with the organization. Channel management included multiple platforms including Social Media with nearly sixteen channels to interact with Emirates ID.

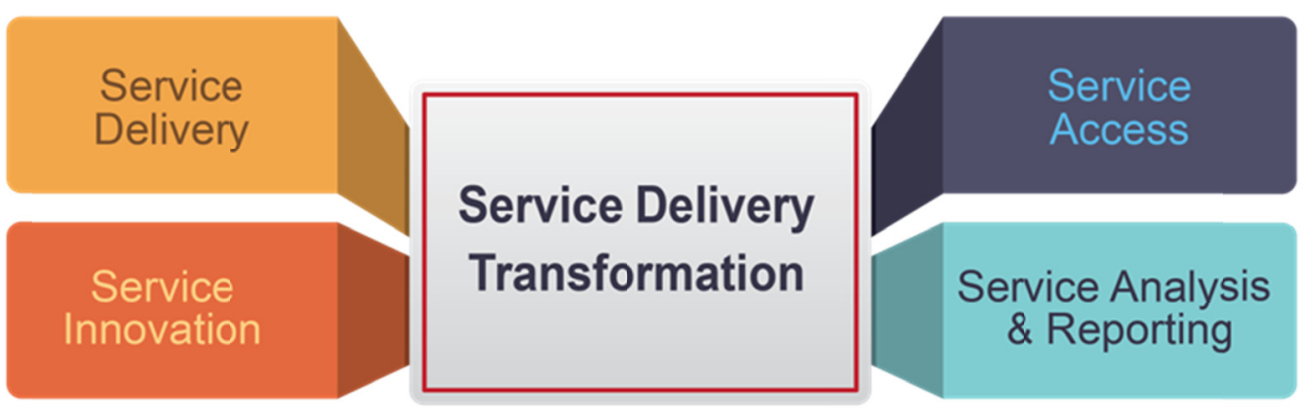

Figure 23. Service Delivery Transformation Model 
The E-Transformation was designed to thus provide Service Delivery Transformation-see Figure 23. The business processes related to service delivery were automated and integrated to provide a seamless delivery mechanism. Service access was enhanced by enabling multiple platforms and channels for communication and interaction with the organization. Organizational innovation was directed toward providing enhanced customer experiences resulting in service innovation. Examples of such service innovation were seen in the deployment of ID-card-renewal services that enabled instant issuance of Digital IDs to the citizens. Service-level management was accorded by smart analysis and online reporting tools.

\subsection{Tier 3: Leadership and Communication}

The third tier of the framework deals with dimensions related to communication and leadership. See Figure 24. Each of these is discussed next.

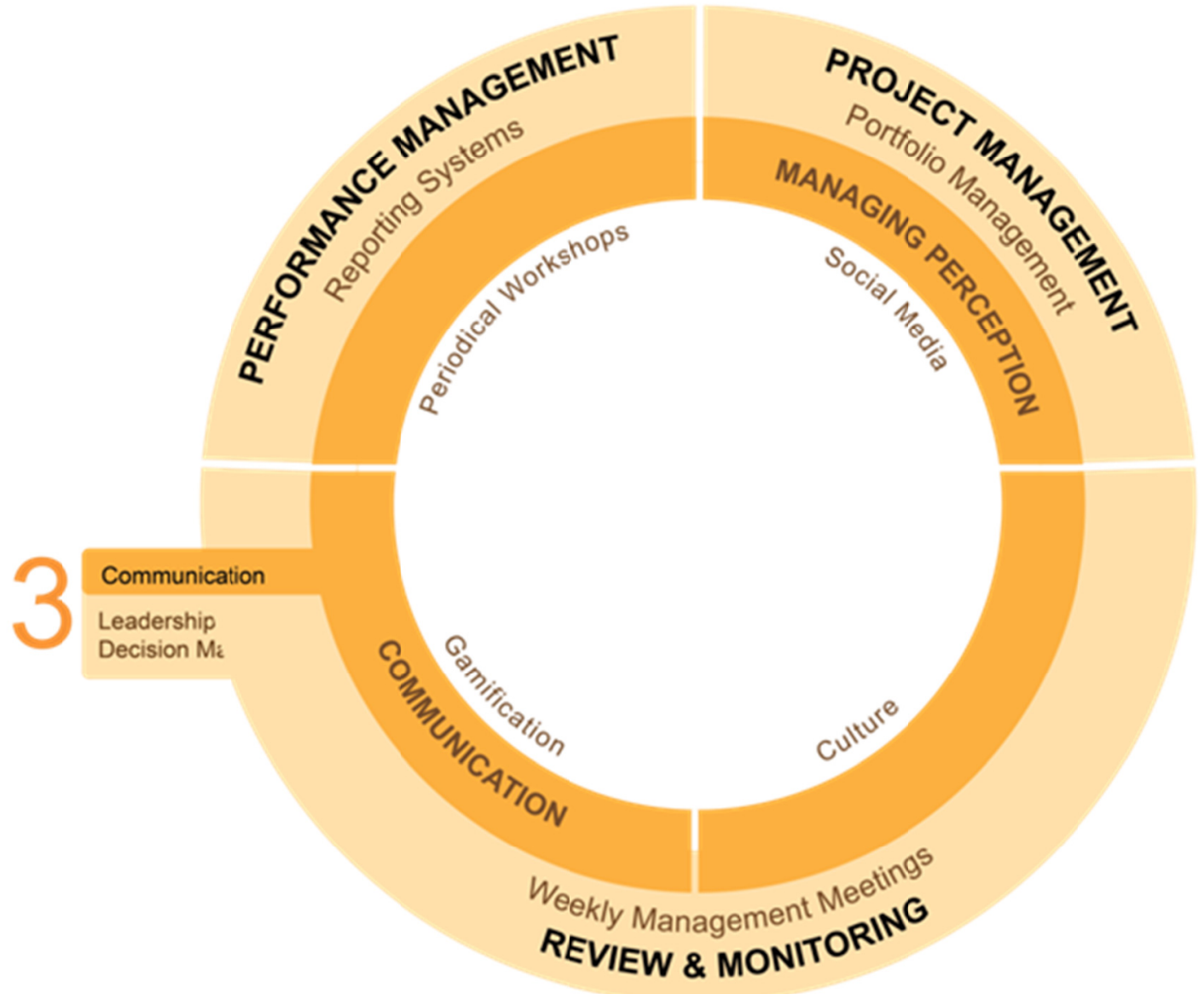

Figure 24. Leadership and Communication Tier

\subsubsection{Communication}

Communication is viewed to be the most important success attribute at Emirates ID. Communication was the thread that established the relationship between all constituents (management, employees, partners, customers, etc.). By focusing on results and relationships, the communication approach generated solid outcomes related to discipline, accountability, strategic alignment, transparency and management of values, and empowerment of employees. With both internal and external audience targets (employees and customers), communication initiatives were results-driven and focused on achieving measurable results as per the targets described by the strategic plan (Organization's Image). See also Figure 25. 


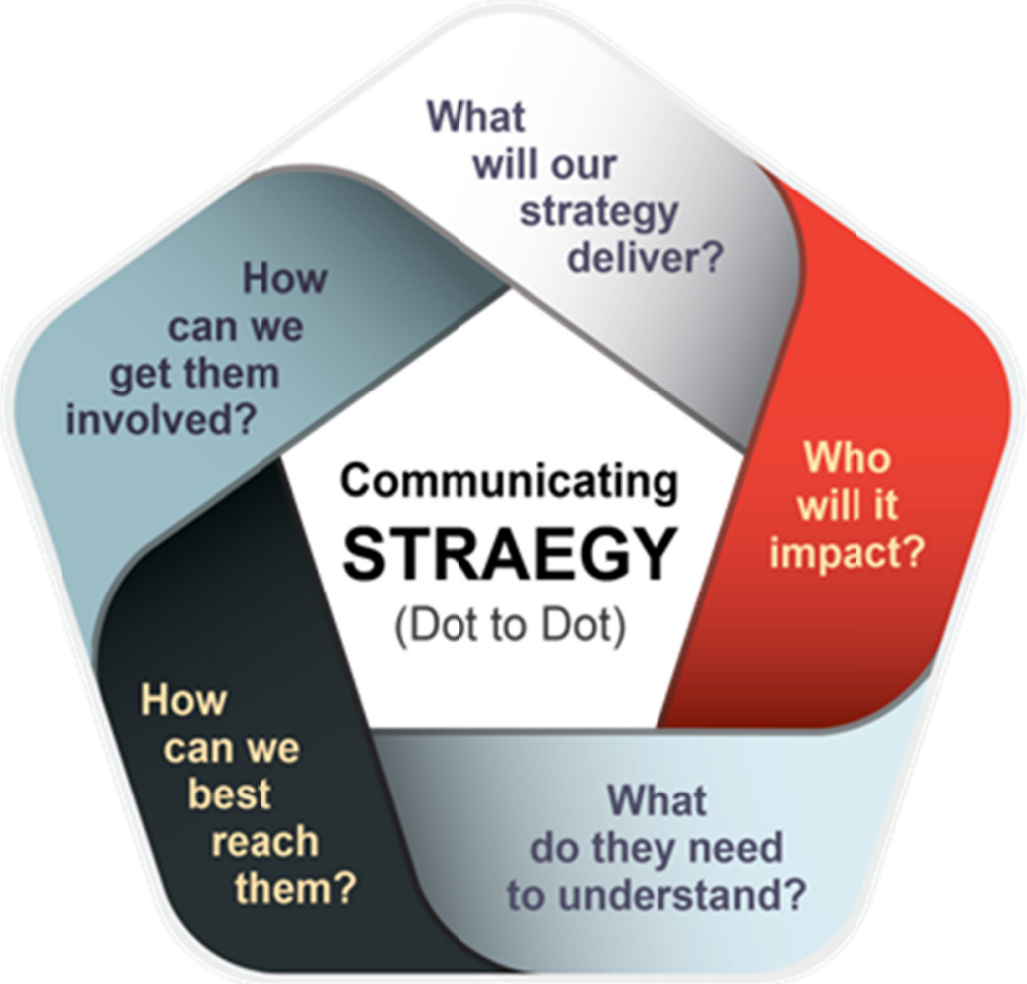

Figure 25. Communication Strategy Questions

The internal communication strategy at Emirates ID was inclusive in nature, in which all employees were given the opportunity to comment on decisions that affected their day-to-day work. Employees' involvement was seen as a key factor that affected employee satisfaction and success at Emirates ID. In order to have a successful strategy execution, all the individuals involved in the process must be fully aware of the strategy from the formulation of the Mission, Vision, and Values to the decisions made in strategic and operational review meetings. Hence, the strategic priorities are continuously communicated internally via different channels.

- Vision, Mission, Values, and Strategy Map were posted on the walls of every one of the Centers in order to spread awareness of the strategy and keep everyone focused in the same strategic direction;

- Our 2010-2013 Strategic Plan was published in a handbook format and distributed to all the employees of the authority: and

- Several workshops and meetings were also held throughout the year to discuss strategy and increase awareness of Emirates ID Authority's strategic priorities.

Regarding external communication, throughout the phases of strategy development and execution, Emirates ID had been always keen to present and discuss its strategic objectives and initiatives with all its partners and stakeholders. Emirates ID aimed to encourage the sharing of knowledge, feedback, and perspectives as it strived, based on its mission, to contribute to national and individual security by establishing personal identities in the UAE and to revolutionize service delivery in both the public and private sectors.

Emirates ID also developed an open communication environment in which employees and customers were able to freely share feedback, ideas, and criticism through different platforms (social media, contact center, SMS, in-house feedback system, etc.). It has developed more than 16 different interactive channels in this regard. Management was committed to open communication as means of building an environment of mutual trust.

\subsubsection{Change Management: Re-Tuning Culture}

The preparation and implementation of change is highly culturally sensitive and is one of the most difficult of the leadership challenges (Denning, 2011; Wursten, 2010). That is because an organization's culture comprises an interlocking set of goals, roles, processes, values, communications practices, attitudes, and assumptions (Denning, 2011). 


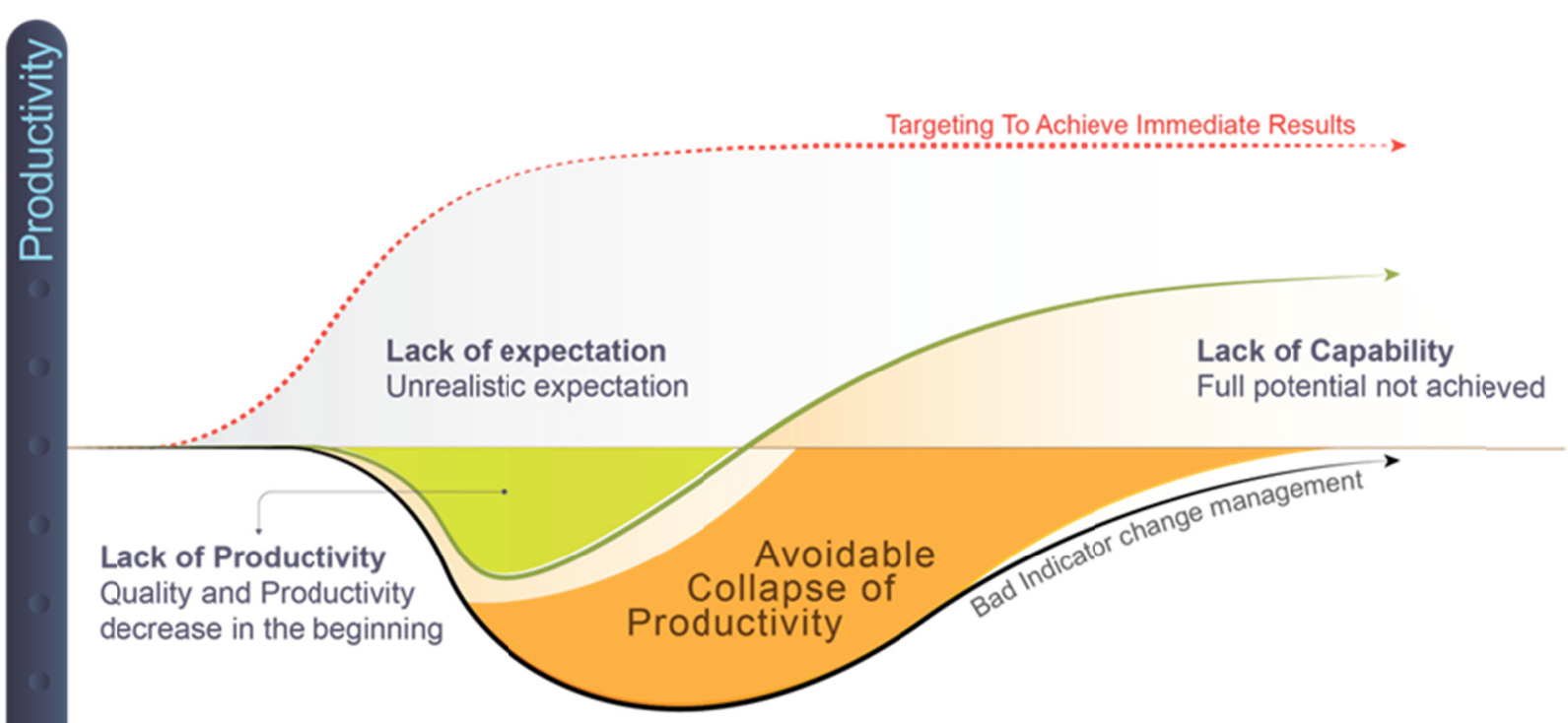

\section{Time}

Figure 26. Change Management

Emirates ID used its own change management methodology that was largely based on Kotter's (1996) 8 Steps of Change Model. (See Appendix-1) The three main dimensions of the methodology included:

(1) Setting the tone: Creating the climate for the change and determining what is changing;

(2) Communication: Preparing and delivering a single message and vision for change; and,

(3) Recognizing and rewarding positive behavior: Implementing and sustaining the change, enabling action, and celebrating short-term wins.

All in all, change management required careful and extensive involvement of top management teams and the utilization of all organizational tools. The order in which they were deployed had a critical impact on the success of such endeavors. See also Figure 27. 


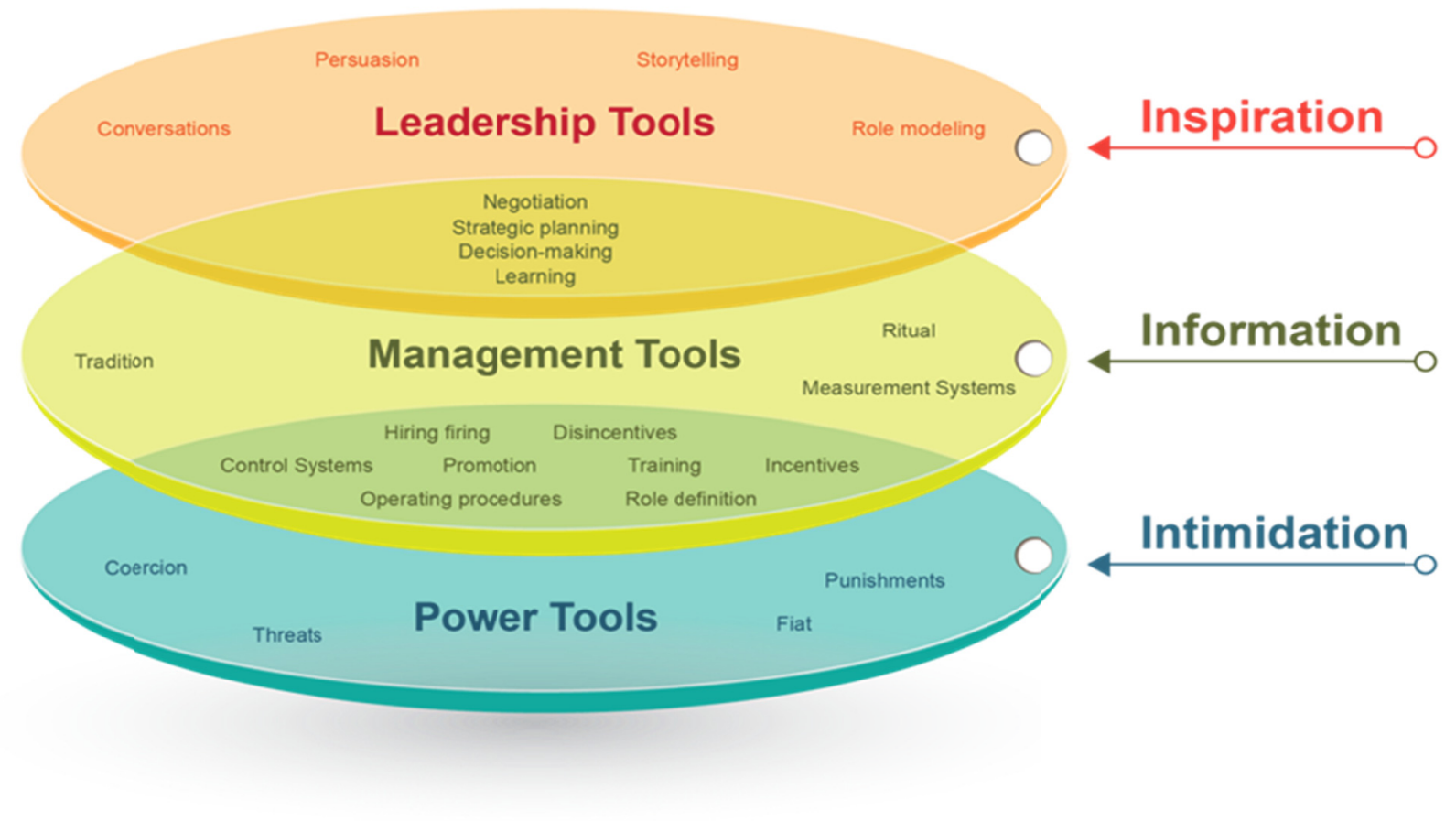

Figure 27. Organizational Tools for Change Management Source: (Gartner, 2014)

\subsubsection{Gamification}

Gamification is the concept of applying game mechanics and game-design techniques to engage and motivate people to achieve their goals (Gartner, 2014) (Note 5). Emirates ID used gamification quite extensively. Emirates ID introduced gamification on different levels that had a significant impact on the overall performance and collaboration between employees. One example of this was the embedding of game-design elements into a variety of virtual and in-person employee and customer-engagement activities.

The Authority also used gamification to capture organizational knowledge in short cartoon videos and passed them to both employees and customers to enhance their awareness of work-related processes and procedures. This proved to be a more effective mechanism to engage people and offer them the opportunity to reflect and refine work elements (Al-Khouri, 2014).

\subsubsection{Engaging Stakeholders: Periodical Meetings}

Emirates ID had to carefully identify its stakeholders and those who had interest or would be potentially affected by the strategy delivery or outputs (employees, customers, suppliers, board of directors, government agencies, etc.). Engagement was ultimately about building ongoing communications and to keep everybody on the same page. The authority had to develop a good understanding of the level of engagement that was being sought. This was broadly categorized into 5 categories as depicted in Table 1. See also Appendix-2 for a high-level engagement model followed at Emirates ID.

Table 1. Levels and Engagement Goals

\begin{tabular}{ll}
\hline Informational & $\begin{array}{l}\text { Provide balanced and objective information to assist stakeholders in understanding } \\
\text { the issues, alternatives, opportunities, and/or solutions. }\end{array}$ \\
$\begin{array}{l}\text { Consultation } \\
\text { Involvement }\end{array}$ & $\begin{array}{l}\text { Obtain feedback on analysis, alternatives, and/or decisions. } \\
\text { Work directly with stakeholders throughout the strategy development and execution } \\
\text { process to ensure that concerns and aspirations are consistently understood and } \\
\text { considered in the strategy. }\end{array}$ \\
Collaboration & $\begin{array}{l}\text { Partner with stakeholders in each aspect of the strategy development, including the } \\
\text { development of alternatives and the identification of the preferred solution. } \\
\text { Empowerment }\end{array}$ \\
& $\begin{array}{l}\text { To empower management and business units to perform business functions with } \\
\text { decision-making capabilities. }\end{array}$ \\
\hline
\end{tabular}


A wide variety of internal and external stakeholders were part of the planning process as the authority actively sought to engage stakeholders, build partnerships, integrate initiatives, and showcase the results. Most importantly, the engagement activities helped define and redefine strategic and operational priorities, assign responsibilities, mitigate risks, establish accountabilities, improve productivity, and resolve conflicts. Nonetheless, engagement was challenging as it required significant communication and commitment from all parties.

\subsubsection{Project Management: Portfolio Management}

Particular attention was given to how projects were managed. A Project Office was formed to monitor projects that reported to the Management Board, while the overall management of projects was left to the appropriate departments. Projects were typically analyzed and prioritized based on their relation to strategy objectives, expected benefits and costs, resource consumption, and other priorities within an organization's wider portfolio of projects. Enterprise project management software was used.

The project-management office provided capabilities for issue resolution and risk mitigation, as well as the centralized visibility to help planning and scheduling teams to identify suitable approaches to deliver projects and programs. Project and Portfolio Management helped the organizations gain visibility and control across all work, enhancing decision-making, improving alignment with business strategy, maximizing resource utilization, and enhancing project execution to optimize ROI.

\subsubsection{Performance Management: Reporting Systems}

Performance management is the process of creating a work environment or setting in which people are enabled to perform to the best of their abilities. Management teams spent sustainable amounts of time in defining, tuning, and re-tuning key performance dashboards. This supported the organization in making daily decisions and addressing challenges as they arose. The primary tool used to support performance management was a Balanced Scorecard.

This supported the organization to align strategic objectives on departmental and individual levels. It facilitated effective delivery of strategic and operational goals by following a very structured framework to develop, implement, and cascade the strategy according to the Best Practice Balanced Scorecard Methodology.

Emirates ID's performance-management approach was applied in a way to drive the organization toward the defined strategic direction and to link it with day-to-day activities. A strategy cascading architecture was conceived to align the different departments of the organization to the corporate strategy. The corporate strategic objectives were cascaded throughout the organization as each department defines its internal objectives based on the strategic objectives of the organization. This structured framework ensured explicit linkages between every level of the strategy, from the vision all the way down to activities; hence providing a coherent road map to deliver Emirates ID's mandate.

\subsubsection{Review \& Monitoring: Management Meetings}

A key factor behind the strategy success at Emirates ID can be represented in top management's commitment to make the strategy a 'perpetual transformational machine.' The higher management met on a frequent basis to discuss performance and organizational issues. These meetings were headed by the Director-General and one of Executive Directors in his absence. At the beginning of each year, Emirates ID's Management Calendar was developed around three major performance review forums:

- Bi-weekly operational review meetings that discuss issues involved in daily operations monitored progress of operational KPIs, projects, and action plans, and promoted continuous improvement.

- On a quarterly basis, strategy review meetings were held to assess and discuss the evolution of the Strategic Plan and the overall performance of the organization. During these meetings, the management team fine-tuned strategic targets and initiatives and made any required midcourse adaptations to the strategic plan.

- Finally, a strategy refresh meeting at the end of each year reviewed overall annual performance of the organization, rethinking the strategic assumptions and updating the Strategy Map \& Balanced Scorecard accordingly.

The main purpose of these management forums was to ensure that strategy execution was proceeding as planned, analyze the reasons behind deviations, provide recommendations to improve performance, and make decisions on corrective actions. The key driver of management decisions within Emirates ID were the recommendations made by managers based on periodical analysis and reporting of performance indicators and initiatives defined at each organizational level. 
A formal reporting process followed every month, where each manager updated the status of the KPIs and Initiatives under his or her responsibility and made a detailed analysis on the evolution and proposed corrective actions for underperforming areas. These analyses and recommendations were consolidated and submitted to the leadership team for prioritization of actions and allocation of resources. This represented the last component in the strategic framework. The next section will depict the results achieved by Emirates ID through the implementation of this framework.

\section{Results}

Some of the remarkable results achieved by Emirates ID are depicted in Table 2.

Table 2. Emirates ID Key Performance Indicators 2009-2013

\begin{tabular}{|c|c|c|c|}
\hline & \multirow{2}{*}{ OUTCOME KEY PERFORMANCE INDICATORS } & \multicolumn{2}{|c|}{ Results } \\
\hline & & 2009 & 2013 \\
\hline$\# 1$ & Key objective attained ( $\%$ of population enrolled) & $17 \%$ & $96 \%(+464 \%)$ \\
\hline \multirow{2}{*}{$\# 2$} & Annual Budget & Government & Self-Sufficient from \\
\hline & Anmual Buaget & Dependent & 2011 \\
\hline \multicolumn{4}{|c|}{ CUSTOMER KEY PERFORMANCE INDICATORS } \\
\hline$\# 1$ & Average waiting time per customer in registration centers & $60 \mathrm{~min}$ & $10 \min (-\mathbf{8 3 . 3} \%)$ \\
\hline \#2 & $\%$ of Customer Satisfaction & $64 \%$ & $88 \%(+\mathbf{3 7 . 5 \%})$ \\
\hline \multicolumn{4}{|c|}{ INTERNAL PROCESSES KEY PERFORMANCE INDICATORS } \\
\hline$\# 1$ & Average registration capacity at registration centers & 3,000 & $26,911(+797 \%)$ \\
\hline \#2 & Average daily registration of residents in the population register & 5,926 & $21,226(+\mathbf{2 5 8} \%)$ \\
\hline \#3 & Average daily Card production volume & 3,636 & $17,103(+370 \%)$ \\
\hline \#4 & Average time required to register a customer & $30 \mathrm{~min}$ & $5(-83.3 \%)$ \\
\hline \#5 & Average service time per customer in registration centers & $90 \mathrm{~min}$ & $15(-\mathbf{8 3 . 3 \%})$ \\
\hline \#6 & Completion of secure infrastructure for data sharing (\%) & $44 \%$ & $61 \%(+17 \%)$ \\
\hline \#7 & $\%$ of procedures automated & $58 \%$ & $67 \%(+9 \%)$ \\
\hline \multicolumn{4}{|c|}{ HUMAN CAPITAL KEY PERFORMANCE INDICATORS } \\
\hline$\# 1$ & Job turnover rate for nationals & $12 \%$ & $5 \%(-58.3 \%)$ \\
\hline \#2 & Employee satisfaction & $57 \%$ & $78 \%(+36.8 \%)$ \\
\hline
\end{tabular}

Based on these results, Emirates ID Authority was selected as the top performer in many organizational practices in the UAE Government Excellence Program for two consecutive cycles of 2012 and 2014. The assessment was based on an EFQM excellence model and was conducted by the UAE Prime Minister's Office. Following are the key categories where Emirates ID was recognized in the Government Excellence Program:

- Best Federal Authority (below 900 employees), 2012

- Best Federal Authority (above 900 employees), 2014

- Best Federal Entity in Customer Care and Service Provisioning, 2012 and 2014

- Best Federal Entity in Organizational Leadership, 2012

- Best Federal Entity in Human Resources Management Practices, 2014

- Best Practices in Partnerships, 2012

- Best Practices in Technical Teams, 2014

- Best Practices in Knowledge Management, 2014

Emirates ID today is recognized as an international benchmark for strategic management, organizational development, and for innovation in core functions and management systems. It was also considered as one of the 
most pioneering government organizations in the world according to the recent United Nations report (UN, 2014). Similar case studies were also prepared by international institutions that illustrated Emirates ID as an example in key areas of organizational excellence, e.g., (INSEAD, 2013; Palladium, 2014; UAE University, 2014).

\section{Closing Notes}

Organizations do not develop by themselves. They need to be nurtured and cared for, enabling them to evolve into mature socially responsible entities. Organizational development is thus about planning and implementation to achieve organizational goals (Bradford and Burke, 2005; Carton and Hofer, 2010; Chhinzer and Ghatehorde, 2009). With a strong and robust Strategy Framework, organizations can select the most adequate management tools and systems and devise specific initiatives and programs to raise themselves from being mediocre to great by accelerating their pace of development (Johansson et al., 1993; Peters, and Waterman, 1992; Prinsloo et al., 1999; Prescott, 1998; Scholtz, 1997). This article attempted to discuss one such successful organization.

In addition to what has been demonstrated in this article, we believe that leadership and communication were primary factors behind the success of the organization. In fact, if we zoom a little into the failure factors reported by the different research studies, we see a common thread linking the majority of factors and relating them to leadership and communication.

Leadership is all about drawing a holistic and overall picture of what the organization needs to achieve. Leaders are those who are expected to get the pieces together when the "what next?" is blurry to everyone else. Higher management at Emirates ID played a key role in the selection and enabling of management teams at all levels to perform their tasks. The higher management acted as a navigational instrument that supported the organization to determine what and where to concentrate effort. In fact, this was a continuous process, as management at operational layers tended to lose focus during execution stages.

Leaders were the ones who supported the organization to focus on what is important and prioritize the projects and activities that contributed to results and not just outputs. Focus was always on the $20 \%$ of organizational work that normally would provide $80 \%$ of value. See also Figure 28 .

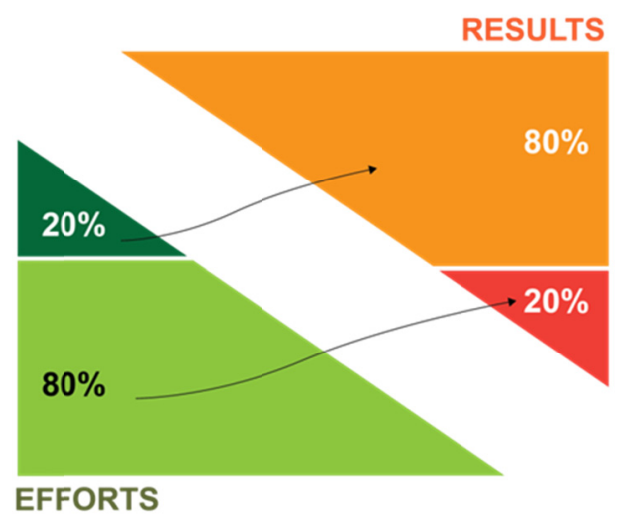

Figure 28. 80-20 Pareto Rule

Communication played a magical role in shaping the culture at Emirates ID. Management's communication style and strategies helped to some extent avoiding cultures of 'passing blames' and 'excuses' and supported the organization to focus on the solutions instead. See also Figure 29. 


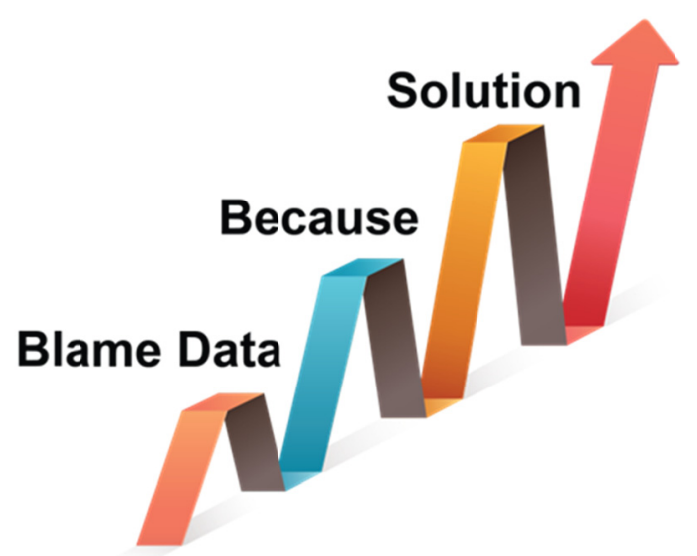

Figure 29. Shifting Individuals' Mindsets to Focus on Solutions

Besides, one of the most important lessons obtained by management at Emirates ID was the "starting from the end." This was not only a stressful management activity, but also a critical success factor that needed to be maintained. In fact, this helped to align expectations with delivery and avoid mismatches in communications. See also Figure 30.

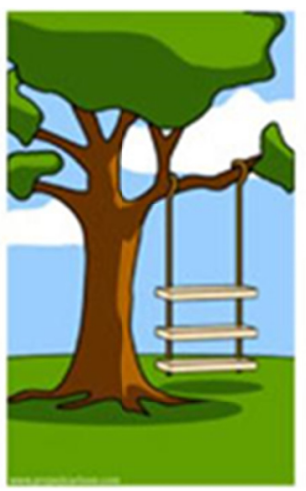

Expectation

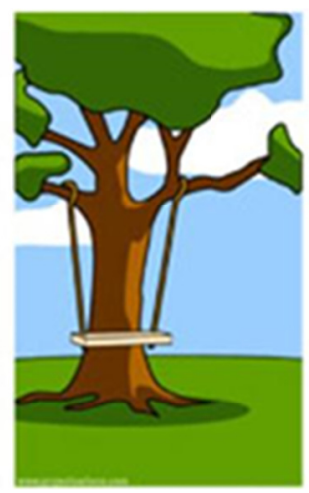

Analysis

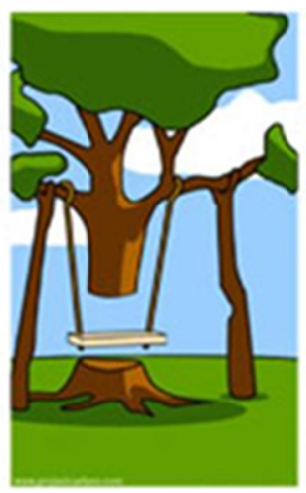

Design

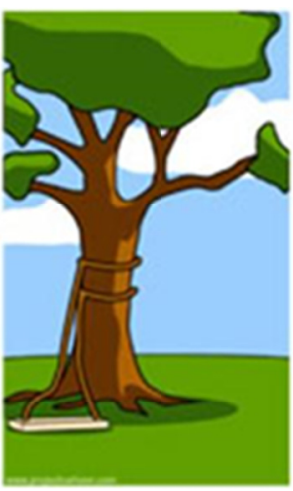

Execution

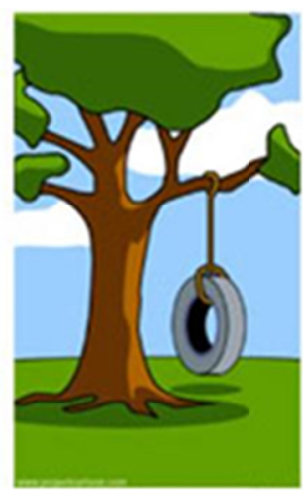

Delivery

Figure 30. Typical Development Stages Leading to Failure in Delivering in Accordance to Expectations

Indeed, there is no one-size-fits-all framework, nor a magic bullet solution to ensure effective strategy execution. Successful strategies are just products of well-designed and executed work. Learning from and building on others' experiences is indeed something that cannot be ignored. The presented strategic organizational development framework provides a successful case study from a public sector. It may serve to provide a more measured than straightforward 'gung ho' development. It can be used to systematically identify organization-wide developmental opportunities. Throughout the world, government entities are under pressure to make efficiency gains while improving the customer experience. Emirates ID provides a best practice case on how this can actually happen in practice: having a clear and structured long-term plan that guides the implementation of management systems, tools, and processes and drives strategic, tactical, and operational decisions. Empirical research to test the application of the framework may contribute to further investigation of its appropriateness for generalizability. This may also open a dialogue for a discussion and the development of a more comprehensive standard for organizational development in the public sector.

\section{Acknowledgements}

The author wishes to express his appreciation to Dr. David Norton and Dr. Robert Caplan from Harvard University for their constructive feedback on this work that helped shape the final content. 


\section{References}

Accenture. (2013). Business \& Operating Model Strategy: Service Overview. Retrieved from http://www.accenture.com/us-en/Pages/service-business-operating-model-strategy.aspx

Al-Khouri, A.M. (2014). Fusing Knowledge Management into Public Sector Corporates Excellence Culture: A Review of the Field and The Case of Emirates Identity Authority. Journal of Knowledge Management, Economics and Information Technology, 4(3), 1-89.

Beckhard, R. (1969). Organization development: strategies and models. Reading, Mass.: Addison-Wesley.

Bradford, D.L., \& Burke, W.W. (Eds.) (2005). Organization development. San Francisco: Pfeiffer, 2005.

Branston, J.R., Tomlinson, P.R., \& Wilson, J.R. (2009). 'Strategic Failure' and the case of the UK's former Building Societies: Lessons for the reform of governance in the UK Banking sector. University of Bath School of Management, Working Paper Series from http://www.bath.ac.uk/management/research/pdf/2009-09.pdf

Carton, R.B., \& Hofer, C.W. (2010). Organizational financial performance: identifying and testing multiple dimensions. Academy of Entrepreneurship Journal, 16(2), 1-22.

Cascio, W., Young, C., \& Morris, J. (1997). Financial Consequences of Employment-Change Decisions in Major U.S. Corporations. Academy of Management Journal, 40(5), 1175-1189. http://dx.doi.org/10.2307/256931

Cespedes, F.V., Dougherty, J.P., \& Skinner, B.S. (2013). How to Identify the Best Customers for Your Business. MIT Sloan Management Review, 54(2), 53-59.

Chapman. J. (2002). System failure: Why governments must learn to think differently, 2nd Edition. Demos, London. Retrieved from www.demos.co.uk/files/systemfailure2.pdf

Chhinzer, N., \& Ghatehorde, G. (2009). Challenging relationships: HR metrics and organizational financial performance. The Journal of Business Inquiry, 8(1), 37-48.

Child, J. (2005). Organization Contemporary Principles and Practice. Malden, MA: Blackwell Publishing.

Cohen. J. (2013). Motivation Is A Muscle: The 7 Best Ways To Substantially Increase Your Productivity. Retrieved from

http://www.forbes.com/sites/jennifercohen/2013/11/06/motivation-is-a-muscle-the-7-best-ways-to-substantially -increase-your-productivity/

Cummings, T.G., \& Worley, C.G. (2001). Organization Development \& Change. Mason, OH: Thomson/South-Western.

Denning, S. (2011). How Do You Change An Organizational Culture? Forbes. Retrieved from http://www.forbes.com/sites/stevedenning/2011/07/23/how-do-you-change-an-organizational-culture/

Devarajan, S., \& Kanbur, R. (2012). The Evolution of Development Strategy As Balancing Market and Government Failure, The World Bank and Cornell University. Retrieved from http://kanbur.dyson.cornell.edu/papers/Devarajan-Kanbur9July2012.pdf

European Commission. (2010). Imp3rove: A European Project with Impact: 50 Success Stories on Innovation Management, $\quad$ European Union. $\quad$ Retrieved from http://www.eurosfaire.prd.fr/7pc/doc/1313739930_nbna24180enc_002.pdf

European Framework for Quality Management - EFQM. (2015). Retrieved from http://www.efqm.org

Freeman, R.E. (1984). Strategic management: A stakeholder approach. Boston: Pitman.

French, W.L., \& Bell, C. (1973). Organization development: behavioral science interventions for organization improvement. Englewood Cliffs, N.J.: Prentice-Hall.

Gartner. (2011). 50 percent of organizations that manage innovation processes will gamify those processes. Retrieved from http://www.gartner.com/newsroom/id/1629214

Gartner. (2014). Gamification. $\quad$ Retrieved from http://blogs.gartner.com/brian_burke/2014/04/04/gartner-redefines-gamification/

Gavan, V. (2012). How to create a customer centric culture, Dynamic Business. Retrieved from http://www.dynamicbusiness.com.au/small-business-resources/growing/how-to-create-a-customer-centric-cultur e-29022012.html 
Grundstein, M. (2007). MGKME, A Model for Global Knowledge Management within the Enterprise. Retrieved from http://michel.grundstein.pagesperso-orange.fr/English/MGKME\%20E.htm

Harrison, L., \& Callan, T. (2013). Key research concepts in politics and international relations (key concepts). London: Sage Publications.

Heeks, R. (2003). Most e-Government-for-Development Projects Fail How Can Risks be Reduced? IDPM. Retrieved from http://unpan1.un.org/intradoc/groups/public/documents/cafrad/unpan011226.pdf

HRN-Europe. (2009). Top 10 reasons why strategies fail. Retrieved from http://www.hrneurope.com/2009/11/top-10-strategy-failures

INSEAD. (2013). Establishing a National ID Programme in the UAE. Retrieved from $\mathrm{http}: / /$ centres.insead.edu/innovation-policy/research/documents/National-ID.pdf

Inspirion. (2014). Being Customer Driven is a Journey. Inspirion Consulting. Retrieved from http://inspirionconsulting.com/overview/

Johansson, H.J., McHugh, P., Pendlebury, A.J., \& Wheeler, W.A. (1993). Business Process Reengineering: BreakPoint Strategies for Market Dominance. New Jersey: John Wiley \& Sons.

Kaplan, R., \& Norton, D. (1996). The balanced scorecard: translating strategy into action. Boston, Mass.: Harvard Business School Press.

Kotter, J.P. (1996). Leading Change. Boston: Harvard Business School Press.

Krick, T., Forstater, M., Monaghan, P., \& Sillanpää, M. (2005). The Stakeholder Engagement Manual, vol. 2: The Practitioner's Handbook on Stakeholder Engagement, 2005. Retrieved from http://www.accountability.org/about-us/publications/the-stakeholder.html

Margulies, N. (1972). Organizational development: values, process, and technology. New York, NY: McGraw-Hill Book Co.

Maslow, A.H. (1943). A theory of human motivation. Psychological Review, 50(4), 370-96. http://dx.doi.org/10.1037/h0054346

Mass, A. van der. (2014). Why Strategies Fail, Strategos Consulting. Retrieved from http://www.slideshare.net/ArnoudvanderMaas1/why-strategies-fail-31535568

Palladium. (2014). Strategy Execution Champions, The Palladium Balanced Scorecard Hall of Fame Report 2014 Retrieved from http://hbr.org

Peters, T.J., \& Waterman, R.H. (1992). In search of excellence. Lessons from America's best-run companies. Chatham: Harper Collins.

Porter, M.E. (1985). The Competitive Advantage: Creating and Sustaining Superior Performance. NY: Free Press.

Prescott, B.D. (1998). Creating a world-class organisation: Ten performance measures of business success. London: Kogan Page.

Prinsloo, K.J., Moropodi, J.N., Slabbert, J.A., \& A.J. Parker, (1999). A perspective on the world-class company. Pretoria: Strat-Excell Pty. Ltd.

Rothwell, W.J., Stavros, J.M., Sullivan, R., \& Sullivan, A. (2010). Practicing organization development: A guide for leading change. San Francisco, CA: Jossey-Bass.

Scholtz, H. (1997). Can your company measure its business excellence? The Edge, 44-46, July.

Thornhill, A., \& Saunders, M. (1998). The Meanings, Consequences and Implications of the Management of Downsizing and Redundancy: A Review. Personnel Review, 27(4), 271-295. http://dx.doi.org/10.1108/00483489810213874

UAE University. (2014). Customer service culture, The Government Summit. Retrieved from http://www.thegovernmentsummit.ae/media/496779/UAE-University-Report_eVersion_ar.pdf

United Nations - UN. (2014). UN Global e-Government Survey 2014. Retrieved from http://unpan3.un.org/egovkb/Portals/egovkb/Documents/un/2014-Survey/E-Gov_Complete_Survey-2014.pdf

Kim W.C., \& Mauborgne R. (2005). Blue ocean strategy: How to create uncontested market space and make the competition irrelevant. Boston, Mass: Harvard Business School Press, 2005. 
Walsham, G. (1995). Interpretive case studies in IS research: nature and method. European Journal of Information Systems, 4(2), 74-81. http://dx.doi.org/10.1057/ejis.1995.9

Western, S. (2010). What do we mean by organizational development. Krakow: Krakow: Advisio Press.

Winston, C. (2006). Government Failure versus Market Failure Microeconomics Policy Research and Government Performance. AEI-Brookings Joint Center for Regulatory Studies, Washington, D.C. Retrieved from http://www.brookings.edu/ /media/research/files/papers/2006/9/monetarypolicy\%20winston/20061003.pdf

Wursten, H. (2010). Culture and Change Management, ITIM International. Retrieved from http://www.itim.org/articleonchangemanagement.pdf

Yin, R. (1993). Applications of case study research. Newbury Park, CA: Sage Publishing.

Zibret, B., Derca, M., \& Miklic, N. (2009). How to become a citizen-centric government, A.T. Kearney. Retrieved from http://www.atkearney.com/documents/10192/6bc77242-149a-4515-b4c7-3295ca1ab594

\section{Notes}

Note 1. Emirates ID's mindset has shifted in the recent years toward achieving employees and customers' happiness rather than the term satisfaction. This required the organization to think and implement more innovate strategies.

Note 2. The EFQM Excellence Model was created in 1991 by the European Foundation for Quality Management (EFQM) as a framework against which applicants for the European Quality Award are judged, and to recognize organizational excellence in European companies. Nowadays, EFQM brings together more than 700 members located in many countries across the world. The EFQM model was developed in 1988 in order to strengthen European companies for global competition. It was last modified in early 2013 to reflect the latest developments and needs of organizations. This international framework of criteria can be applied to all sectors and is used by more than 30,000 organizations worldwide to improve their performance and assess their process toward 'excellence.'

Note 3. A value chain is a set of activities that an organization carries out to create value for its customers. Porter proposed a general-purpose value chain that organizations can use to examine all of their activities, and see how they're connected (Porter, 1985). The way in which value-chain activities are performed determines costs and affects profits, so this tool can help organizations identify potential value sources.

Note 4. IMP $^{3}$ rove $^{\circledR}$ is a unique approach to improving innovation-management performance with sustainable impact. It is an initiative of the European Commission to enhance the innovation capabilities of small- and medium-sized enterprises. See also European Commission (European Commission, 2010).

Note 5. Gartner Group predicts that by 2015, gamification will become as important as Facebook, eBay, or Amazon, and more than $70 \%$ of Global 2000 organizations will have at least one gamified application (Gartner, 2011; Gartenr, 2014).

\section{APPENDIX-1}

Kotter's (1996) 8-step change model was used as a high-level guiding tool for change management at Emirates ID. The model starts by creating a sense of urgency around a changed idea and moves across various stages of the process, ending up with the institutionalization of the change. 


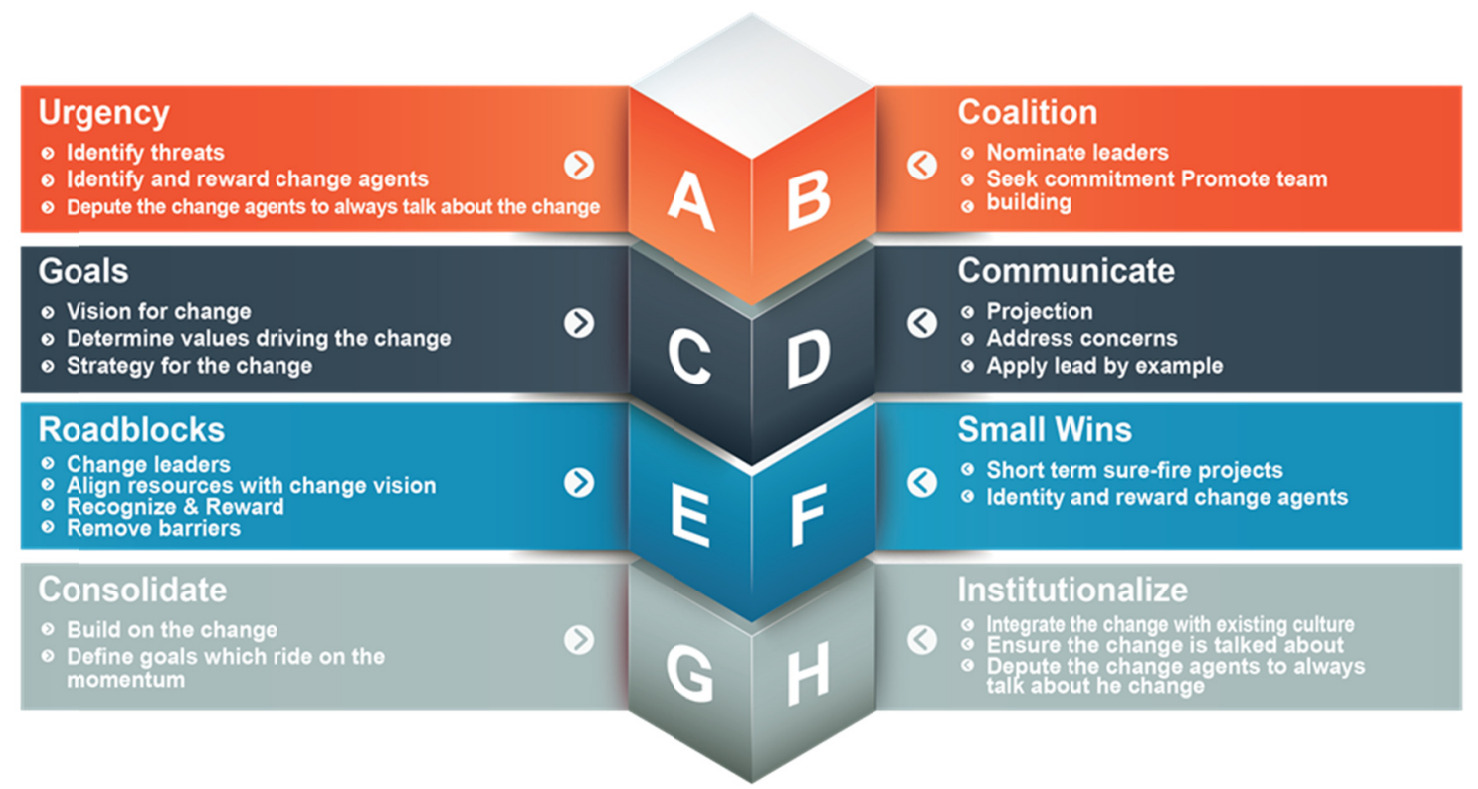

\section{APPENDIX-2}

Figure A-1: Kotter's 8-Step Change Model

Effective stakeholder engagement enables better-planned and more-informed decision-making. It is considered as an essential ongoing activity to align corporate strategy and address complex organizational issues and challenges (Freeman, 1984; Krick, 2005). As such, Emirates ID worked constantly to involve all groups considered to affect or be affected by its new strategy. Figure A-2 depicts the general components of the adopted Stakeholder Engagement Framework. The framework emerged as a vital instrument to foster dialogue and collaboration and to add value and viability to operations based on the needs of all stakeholders.

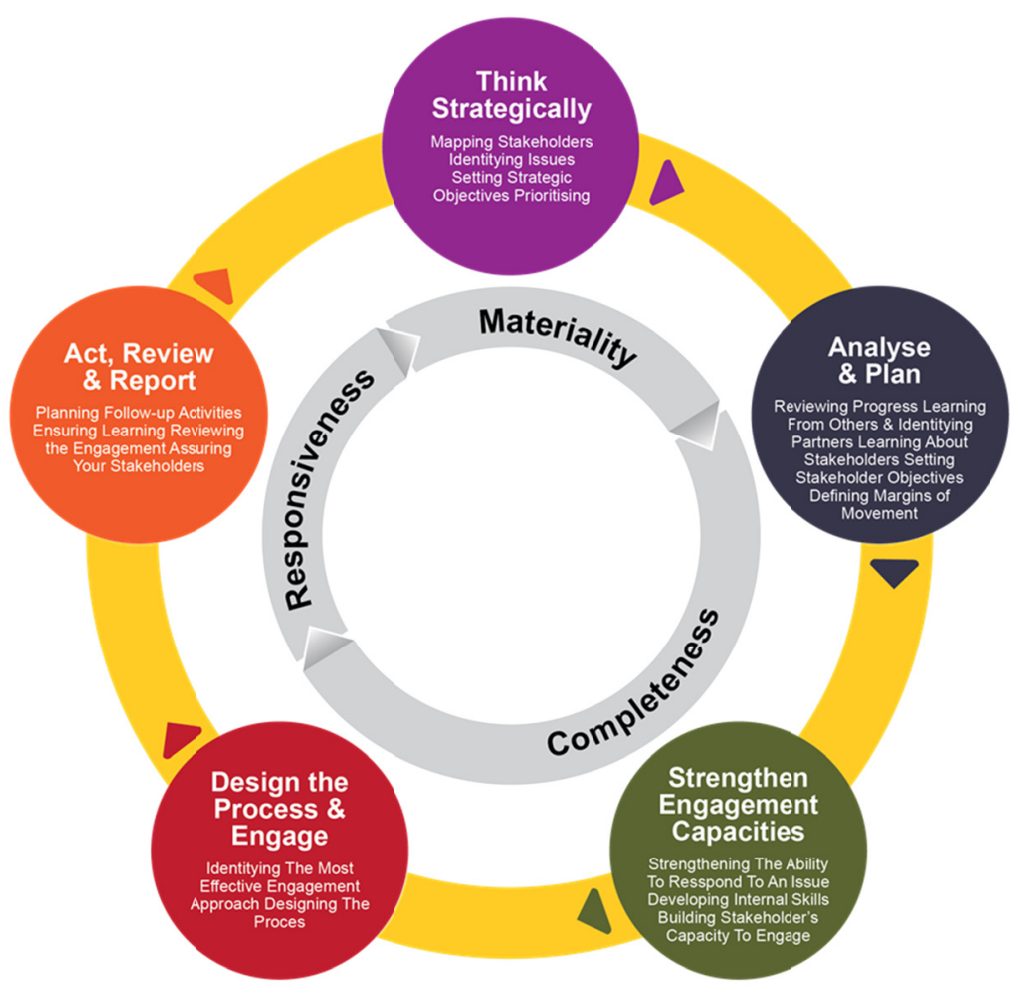

Figure A-2: Stakeholder Engagement Framework Source: (Feeman, 2005) 